\title{
Building eco-surplus culture among urban inhabitants as a novel strategy to improve finance for conservation in protected areas
}

\author{
Minh-Hoang Nguyen 1,2,* \\ Thomas E. Jones ${ }^{1}$ \\ ${ }^{1}$ Graduate School of Asia Pacific Studies, Ritsumeikan Asia Pacific University, Beppu, Oita \\ 874-8577, Japan \\ ${ }^{2}$ Centre for Interdisciplinary Social Research, Phenikaa University, Yen Nghia Ward, Ha Dong \\ District, Hanoi 100803, Vietnam \\ *Correspondence: ng19m6tk@apu.ac.jp (M.-H.N) \\ $* * * * *$
}

\begin{abstract}
The rapidly declining biosphere integrity, representing one of the core planetary boundaries, is alarming. One of the most widely accepted measures to halt the rate of biodiversity loss is to maintain and expand protected areas that are effectively managed. However, it requires substantial finance derived from nature-based tourism, specifically visitors from urban areas. Using the Bayesian Mindsponge Framework (BMF) on 535 Vietnamese urban residents, the current study examined how their biodiversity loss perceptions can affect their willingness to pay for the entrance fee and conservation in protected areas. We found that perceived environmental degradation, loss of economic growth, loss of nature-based recreation opportunity, and loss of knowledge as consequences of biodiversity loss has indirect effects on paying willingness through the mediation of the attitude towards conservation. Especially, the perceived knowledge loss also has a direct positive influence on the willingness to pay for the entrance fee and conservation. In contrast, perceived loss of health is negatively associated with the attitude towards conservation. Based on these findings, we suggest that building an eco-surplus culture among urban residents can be a promising way to generate more finance from nature-based tourism for conservation in protected areas and ease the domestic government's and international organizations' funding allocations problems.
\end{abstract}

Keywords: biodiversity conservation, nature-based tourism, urban residents, sustainable finance, Bayesian Mindsponge Framework, Mindsponge mechanism

\section{Introduction}

Among nine planetary boundaries, which helps define "safe operating space" for human societies development without driving the Earth system away from a Holocene-like condition, climate change and biosphere integrity (measured by the rate of biodiversity loss) are two core boundaries [1]. Despite the vital roles of biosphere diversity in the Earth system, the biodiversity loss rate is occurring at an unprecedented rate. Around 1 million species are threatened with extinction, according to the Intergovernmental Science-Policy Platform on Biodiversity and Ecosystem Services [2]. Moreover, the global numbers of mammals, birds, fishes, plants also dreadfully dropped by 68\% from 1970 to 2016 [3]. To curb the substantial degradation of biological diversity, 
keeping and expanding protected areas are suggested as major solutions. Nevertheless, a substantial amount of finance is needed to maintain the effective operations of such protected areas; otherwise, the "paper park" problem will be rampant [4-6]. Therefore, the current study aims to investigate how biodiversity loss perceptions among Vietnamese urban residents can potentially contribute to conservation initiatives and the finance of protected areas, and in turn, suggest implications for policymakers and protected managers to improve conservation effectiveness.

The past several decades have seen the profound development and expansion of protected areas worldwide in geography and function [7]. Since the first establishment of the world's first national park - Yellowstone national park - in 1872, the total area of protected areas and other effective area-based conservation measures (OECMs) have covered at least 16.64\% (22.5 million $\mathrm{km} 2$ ) of land and inland water ecosystems, and $7.74 \%$ (28.1 million $\mathrm{km} 2$ ) of coastal waters and the ocean [8]. The areas of particular importance for biodiversity and ecosystem services have been increasingly covered, with $65.5 \%$ of Key Biodiversity Areas partially or fully protected [8]. Along with the geographical expansion, protected areas' functions have also been diversified to achieve various conservation, social and economic targets [7]. Due to protected areas' vital roles, effective management and expansion of protected areas over terrestrial and marine areas are set as Target 11 of the Aichi Biodiversity Targets as well as Goals 14 and 15 of the United Nations' Sustainable Development Goals.

Either effective management or expansion of protected areas requires a substantial, sustainable amount of finance. Even though the coverage of protected areas is increasing, inadequate financial support for protected areas is falling behind, leading to poor management, especially in developing countries [9]. In Vietnam, national parks receive funding from the province and national government for full operations and maintenances, while conservation management budget may also come from international donors, such as World Wide Fund for Nature (WWF), Fauna \& Flora International (FFI), International Labour Organization (ILO), etc. Nonetheless, there remain many constraints. Domestic government's subsidies are widespread but insufficient and lack of priority, whereas international aids are large but can only focus on large, site-specific projects [10]. Solely assuring the operation and conservation finance within the park is inadequate to biodiversity conservation, as local people are reliant on the protected areas' resources for livelihood. As a result, tourism is endorsed by many scientists as a sustainable financing source for biodiversity conservation in protected areas $[11,12]$.

The demand for nature-based tourism is one of the fundamental purposes that drive people to visit protected areas. The revenue generated from the influx of visitors to protected areas is massive. On the global scale, Balmford, et al. [13] estimate that around 8 billion visits are made per year to the world's terrestrial protected areas. These visits generate roughly $\$ 600$ billion per year in direct in-country expenditure $\$ 250$ billion per year in consumer surplus. Thanks to the income generated by tourism expenditure, many national parks can pay more than $50 \%$ of their expenditure for park operation and conservation of some endangered species $[9,14]$. Moreover, if the benefits of tourism are allocated in fair and equitable ways, tourism development also helps sustain the local livelihood, which reduces the pressure on conservation efforts [15-17]. 
One of the most widely used methods to generate revenue from visitors within the protected area is levying the fee. Such fees can appear under various forms, like fee within a tour, entrance fee, conservation fee, user fee, etc. [5,12]. Visitors' willingness to pay for the fee is distinct depending on the protected areas' features and the visitors' characteristics [18-22]. Specifically, income level, educational attainment, and institutional trust are strong predictors of increasing willingness to pay for the entrance fee in Dalai Lake protected area [18]. Visitors are more willing to pay more for the protection of Ethiopian wolves if the wolf population increases [20]. However, studies also show that a certain number of visitors are not willing to pay because they attribute biodiversity conservation to the government's responsibility $[18,19]$. Most of the studies regarding willingness to pay are conducted on-site with visitors visiting the protected areas. Therefore, one question arises: "could we improve the visitors' willingness to pay for entrance fees and conservation?" We think that there is, and it is, to improve the willingness to pay among increasing urban residents potential visitors to protected areas, besides international visitors [23-26].

Nevertheless, how could the willingness to pay among urban residents be improved? We hypothesize that the willingness to pay for entrance fee and conservation can be improved through building eco-surplus culture among urban residents. Eco-surplus culture is the term coined by Vuong [27],[28] to indicate the culture that values the protection and healing of nature. In other words, eco-surplus culture is a set of pro-environmental norms, practices, and values that aim to reduce negative anthropogenic impacts on environments as well as conserve and restore nature. The concept is suggested as the $11^{\text {th }}$ element, complementing Harrison [29]'s ten progressive cultural values. In this study, the positive attitude towards conservation can be considered as a representative value of the eco-surplus culture. Nguyen and Jones [30] indicate that perceived consequences of biodiversity loss, such as environmental degradation, losses of economic growth, nature-based recreation opportunities, health, and knowledge, are positively associated with the attitude towards the prohibition of wildlife consumption. Therefore, it is also possible that urban residents' biodiversity loss perceptions are positively associated with their attitude towards conservation, and thus eco-surplus culture. Further explanations of the relationships between biodiversity loss perceptions, conservation-related attitude, and willingness to pay are shown in the Model Construction sub-section.

To our knowledge, little is known about the willingness to pay among urban residents and its predictors. Thus, the current study employed the Bayesian Mindsponge Framework (BMF) to examine the link between perceptions towards biodiversity loss, attitude towards conservation, and willingness to pay for entrance fees and conservation in protected areas among 535 inhabitants in Vietnam's largest cities. The BMF combines the Mindsponge framework as a foundation for model construction and Bayesian inference as an analytical approach to estimate the constructed models.

\section{Method and Materials}

\section{Study Site and Samples}

Using the dataset of Nguyen [31] the current study examined the associations between perceptions towards biodiversity loss and willingness to pay for the entrance fee and conservation among Vietnamese urban residents. The dataset was systematically designed and generated through four 
main steps: 1) questionnaire design, 2) survey collection, 3) data check and validation, and 4) dataset generation.

As there was limited knowledge regarding the perceptions of biodiversity and biodiversity loss in Asian context in general and Vietnam in particular, in-depth interviews were initially conducted with 38 inhabitants in Ho Chi Minh and Hanoi capital cities. The participants' profiles (e.g. gender, age, occupation, etc.) were purposively selected to ensure the diversity of opinions. Nguyen [31] also applied the 'theoretical saturation' principle to determine when to stop the interview process [32]. Based on the responses of 38 people, the questionnaire was designed.

From June 18 to August 8 2021, the questionnaire was distributed through a Web-based survey via Google Forms using the snowball sampling strategy. People living in urban areas were intentionally targeted. The participants were asked to read and agree with a consent form explaining the questionnaire's contents, purposes, and the confidentiality of respondents. Finally, 581 responses were acquired.

Next, a four-step quality check was performed to remove ineligible samples. To elaborate, respondents with residency in non-urban areas, age less than 18, duplicate emails, and poor-quality answers were excluded. After the validation, 535 samples remained. Finally, the dataset was generated and saved under comma-separated value format for easing later uses. The dataset was peer-reviewed by two referees and made available on the open repository for later reproduction, validation, and transparency. More details of the dataset can be found here: https://doi.org/10.11922/sciencedb.j00104.00097.

In this study, we employed eight variables that can be categorized into three main groups. The first group includes five variables demonstrating how urban people perceive the consequences of biodiversity loss in five aspects: 1) environmental degradation, 2) loss of economic growth, 3) loss of nature-based recreation opportunities, 4) loss of health, and 5) loss of knowledge. These five variables were generated from ten variables in the dataset. Some variables are relatively similar, so we grouped them into one variable and took the average value. Specifically, perceived pollution and climate change as consequences of biodiversity loss were grouped into EnvironmentalDegradation, with 0.88 of Cronbach alpha; perceived loss of green space, natural aesthetics, and nature-based recreation were grouped into NatureRecreationLoss, with 0.85 of Cronbach alpha; perceived reduction of physical health, mental health, and life expectancy were grouped into HealthLoss, with 0.92 of Cronbach alpha; EconomicGrowthLoss and KnowledgeLoss remained the same (see Table 1).

Table 1: Variable description

\begin{tabular}{|c|c|c|c|}
\hline Variable & Meaning & $\begin{array}{c}\text { Type of } \\
\text { variable }\end{array}$ & Value \\
\hline EnvironmentalDegradation & $\begin{array}{c}\text { Whether the respondent perceives } \\
\text { environmental degradation } \\
\text { (pollution and climate change) as } \\
\text { a consequence of biodiversity } \\
\text { loss }\end{array}$ & $\begin{array}{c}\text { Ranging from } \\
1 \text { (strongly } \\
\text { disagree) to } 4 \\
\text { (strongly } \\
\text { agree) }\end{array}$ \\
\hline
\end{tabular}




\begin{tabular}{|c|c|c|c|}
\hline EconomicGrowthLoss & $\begin{array}{l}\text { Whether the respondent perceives } \\
\text { the loss of economic growth as a } \\
\text { consequence of biodiversity loss }\end{array}$ & Numerical & $\begin{array}{l}\text { Ranging from } \\
1 \text { (strongly } \\
\text { disagree) to } 4 \\
\text { (strongly } \\
\text { agree) }\end{array}$ \\
\hline NatureRecreationLoss & $\begin{array}{l}\text { Whether the respondent perceives } \\
\text { the loss of naturebased recreation } \\
\text { (loss of green space, natural } \\
\text { aesthetics, naturebased } \\
\text { recreation) as a consequence of } \\
\text { biodiversity loss }\end{array}$ & Numerical & $\begin{array}{l}\text { Ranging from } \\
1 \text { (strongly } \\
\text { disagree) to } 4 \\
\text { (strongly } \\
\text { agree) }\end{array}$ \\
\hline HealthLoss & $\begin{array}{l}\text { Whether the respondent perceives } \\
\text { the loss of health (reduction of } \\
\text { physical health, mental health, } \\
\text { and life expectancy) as a } \\
\text { consequence of biodiversity loss }\end{array}$ & Numerical & $\begin{array}{l}\text { Ranging from } \\
1 \text { (strongly } \\
\text { disagree) to } 4 \\
\text { (strongly } \\
\text { agree) }\end{array}$ \\
\hline KnowledgeLoss & $\begin{array}{l}\text { Whether the respondent perceives } \\
\text { the loss of knowledge as a } \\
\text { consequence of biodiversity loss }\end{array}$ & Numerical & $\begin{array}{l}\text { Ranging from } \\
1 \text { (strongly } \\
\text { disagree) to } 4 \\
\text { (strongly } \\
\text { agree) }\end{array}$ \\
\hline Conservation & $\begin{array}{l}\text { Whether the respondent supports } \\
\text { conservation as a preventive } \\
\text { measure of biodiversity loss }\end{array}$ & Binary & $\begin{array}{c}\text { Agree }=1 \\
\text { Disagree }=0\end{array}$ \\
\hline WillingEntranceFee & $\begin{array}{l}\text { Whether the respondent is willing } \\
\text { to pay for the entrance fee when } \\
\text { visiting protected areas }\end{array}$ & Binary & $\begin{array}{c}\text { Agree }=1 \\
\text { Disagree }=0\end{array}$ \\
\hline WillingDonation & $\begin{array}{l}\text { Whether the respondent is willing } \\
\text { to pay for the entrance fee when } \\
\text { visiting protected areas }\end{array}$ & Binary & $\begin{array}{c}\text { Agree }=1 \\
\text { Disagree }=0\end{array}$ \\
\hline
\end{tabular}

The second group only has one variable that indicates the respondents' attitude towards conservation as a preventive measure of biodiversity loss. The last group consists of two variables implying the willingness to pay for entrance fee and willingness to donate for conservation if the respondents have a chance to visit protected areas.

\section{Model Construction}

The BMF, which combines the ability to explain psychological complexity in the human mind and the statistical advantages of Bayesian, was employed as the method in our study [33]. This analytical approach has been found effective in investigating various psychological phenomena, such as attitude towards biodiversity loss preventive measure, suicidal ideation, book-reading interest, etc. [30,34-36]. In this study, models were initially constructed based on the Mindsponge information processing mechanism to examine how perceptions towards biodiversity loss may affect the willingness to pay for the entrance fee and conservation through the support of conservation as a preventive measure [37,38]. 
According to the Mindsponge mechanism, an individual has a mindset, or a set of core values, that influences thinking, attitudes, and behaviors. For information to enter the mindset, it has to pass through the multi-filtering system. The filtering system consists of two major components: 1) costbenefit judgements and 2) trust evaluation. These two components determine whether to accept, reject, or keep the information in the buffer zone for later use or assessment. Both the cost-benefit judgements and trust evaluation are operated based on the preferences of the mindset and perceived information from the environment [34].

Grounded on the cost-benefit judgements of the mechanism, we assumed that an individual that perceives the adverse effects of biodiversity loss would accept information associated with preventive measures (here is conservation) to enter the mindset. The associations between biodiversity perceptions and support for conservation are, therefore, expected to be positive. When ideation of support for conservation emerges in the mindset, it would subsequently affect the filtering system and accept information involved with conservation to enter mindset. Among conservation-related methods, paying for the entrance fee and donating for biodiversity conservation in protected areas might be perceived as two common ways to support conservation. Therefore, urban residents' recognition of adverse impacts of biodiversity loss might positively affect their willingness to pay for entrance fee and conservation through improving support for conservation measures.

To check our assumptions, we construct the following models. Model 1 examines the associations between perceived consequences of biodiversity loss and support for conservation as a preventive measure among urban residents. Models $2 \mathrm{a}$ and $2 \mathrm{~b}$ estimate influences of the participants' support for conservation on their willingness to pay for the entrance fee and conservation, respectively. Finally, Model 3a and 3b are constructed to check whether the relationships between biodiversity loss perceptions and willingness to pay are also direct associations or only indirect through the pathway of support for conservation. If the direct associations are not confirmed, our assumptions using an information processing mechanism to explain the phenomena can be deemed trustworthy.

Model 1: Conservation $\sim$ EnvironmentalDegradation + EconomicGrowthLoss + NatureRecreationLoss + HealthLoss + KnowledgeLoss

Model 2a: WillingEntranceFee $\sim$ Conservation

Model 2b: WillingDonation $\sim$ Conservation

Model 3a: WillingEntranceFee $\sim$ Conservation + EnvironmentalDegradation + EconomicGrowthLoss + NatureRecreationLoss + HealthLoss + KnowledgeLoss

Model 3b: WillingDonation $\sim$ Conservation + EnvironmentalDegradation + EconomicGrowthLoss + NatureRecreationLoss + HealthLoss + KnowledgeLoss 


\section{Analytical Approach}

The constructed models were then analyzed using Bayesian inference aided by the Hamiltonian Monte Carlo algorithm. Reasons for selecting Bayesian inference as the statistical method are several. First of all, it has a great fit with models constructed using the Mindsponge mechanism [33]. One of the natural advantages of Bayesian analysis is that it treats all properties (including unknown variables) probabilistically. When applied with models constructed based on theoretical foundation, Bayesian analysis helps researchers avoid adding control variables and focus entirely on the theoretically selected variables, ensuring the parsimony principle (or Occam's razor) [39]. Moreover, by helping avoid controlling other variables to reduce the model's residual, Bayesian inference also help reduce the risk of non-reproducibility due to scientific misconduct, like stargazing, $p$-hacking, HARKing, etc. [40,41]

The data analyzed in this study were not randomly sampled, and its size was modest. Still, the Bayesian analysis can complement this weakness as "Bayesian statistics is not based on large samples (i.e., the central limit theorem) and hence may produce reasonable results even with small to moderate sample sizes, especially when strong and defensible prior knowledge is available" [42]. The Mindsponge framework can defend prior selection in the current study. The Hamiltonian Monte Carlo algorithm also helps models get rid of the symmetry assumption dependence [43,44].

Psychological and social sciences are currently facing the reproducibility crisis that results generated using conventional approaches cannot be reproduced [45,46]. Besides the scientific misconduct, Halsey, et al. [47] also imply that the crisis is attributable to the wide sample-to-sample variability of the $p$-value. Understandably, reproducibility crisis is not only the result of method inadequacy but also the rapidly changing society. Due to the widespread Internet and ever-growing transportation, humans are exposed to a substantial amount of information every day, so their mindset, perceptions, attitudes, and behaviors are continuously updated and gradually distinct from what they were years ago [37]. With its belief updating philosophy (through priors), the Bayesian inference can improve the estimation's precision [42].

The use of priors in model fitting is a prime demonstration of Bayesian inference's updating philosophy. Incorporating priors give the researchers to integrate their prior beliefs (based on a theoretical framework, previous empirical evidence, intuition, etc.) into model estimation with current evidence (or likelihood). In the constructed models, variables of biodiversity perceptions have high correlation levels, possibly leading to multicollinearity. The problem can be solved by alleviating weak data identification problems if priors are incorporated into model fitting [48-50]. Here, we set prior distributions of parameters as a normal distribution with mean at 1 and standard deviation at 0.5 , representing our beliefs that all studied associations are positive. In addition, the prior-tweaking technique can also be employed to test the sensitivity of the posterior distributions if prior beliefs are changed [51]. If the posteriors only change slightly when we use norm $(0,0.5)$ as priors representing our disbeliefs on the associations, the results can be deemed robust.

All the Bayesian linear regression analyses were conducted using the bayesvl R package [52]. The package offers researchers a user-friendly and intuitive protocol, the ability to visualize beautiful graphics, and cost-effectiveness [53,54]. Model fitting was conducted with a set of four Markov chains with 5000 iterations for each chain. The first 2000 iterations were installed as a warmup 
period. After the simulation process, the models' goodness-of-fit with the data at hand were validated using the Pareto smoothed importance-sampling leave-one-out cross-validation (PSISLOO) [55]. If the model has a good fit with the data, we would continue checking whether the Markov property, or the convergence of the Markov chains, was held after the simulation process. Effective sample size and Gelman shrink factor are two diagnostic statistics of the Markov property. Apart from them, the property can also be diagnosed visually using the trace, autocorrelation, and Gelman plots. Interpretive details of the diagnoses are presented in the Results section.

\section{Results}

The Bayesian linear regression analysis was conducted on 535 Vietnamese urban inhabitants to examine five models proposed in the Model Construction subsection, and the results are presented in this section. More than half of the respondents were female $(57.08 \%)$ and obtained an undergraduate degree as the highest educational level (61.68\%). Most of the respondents belonged to the age group ranging from 23 to $40(47.11 \%) .85 .63 \%$ of the participants reported that they spent most of their lifetime in urban areas, while the percentages of suburban and rural areas were $10.38 \%$ and $3.79 \%$, respectively. Regarding the willingness to pay, $97.57 \%$ were willing to pay for the entrance fee, and $94.95 \%$ were willing to donate to conservation projects when visiting a protected area in the future.

Model 1: Effects of biodiversity loss perceptions on conservation-related attitude

Model 1 was estimated to examine the associations between biodiversity loss perceptions and conservation-related attitude among urban residents. Five predictor variables used in the model correspond with five different perceptions on the consequences of biodiversity loss: environmental degradation, loss of economic growth, loss of nature-based recreation opportunity, loss of health, and loss of knowledge. PSIS-LOO test was initially performed to check whether Model 1 had a good fit with the collected data. All the $k$-values in Figure 1 are below the 0.5 thresholds, so the model can be considered fit with the data. 


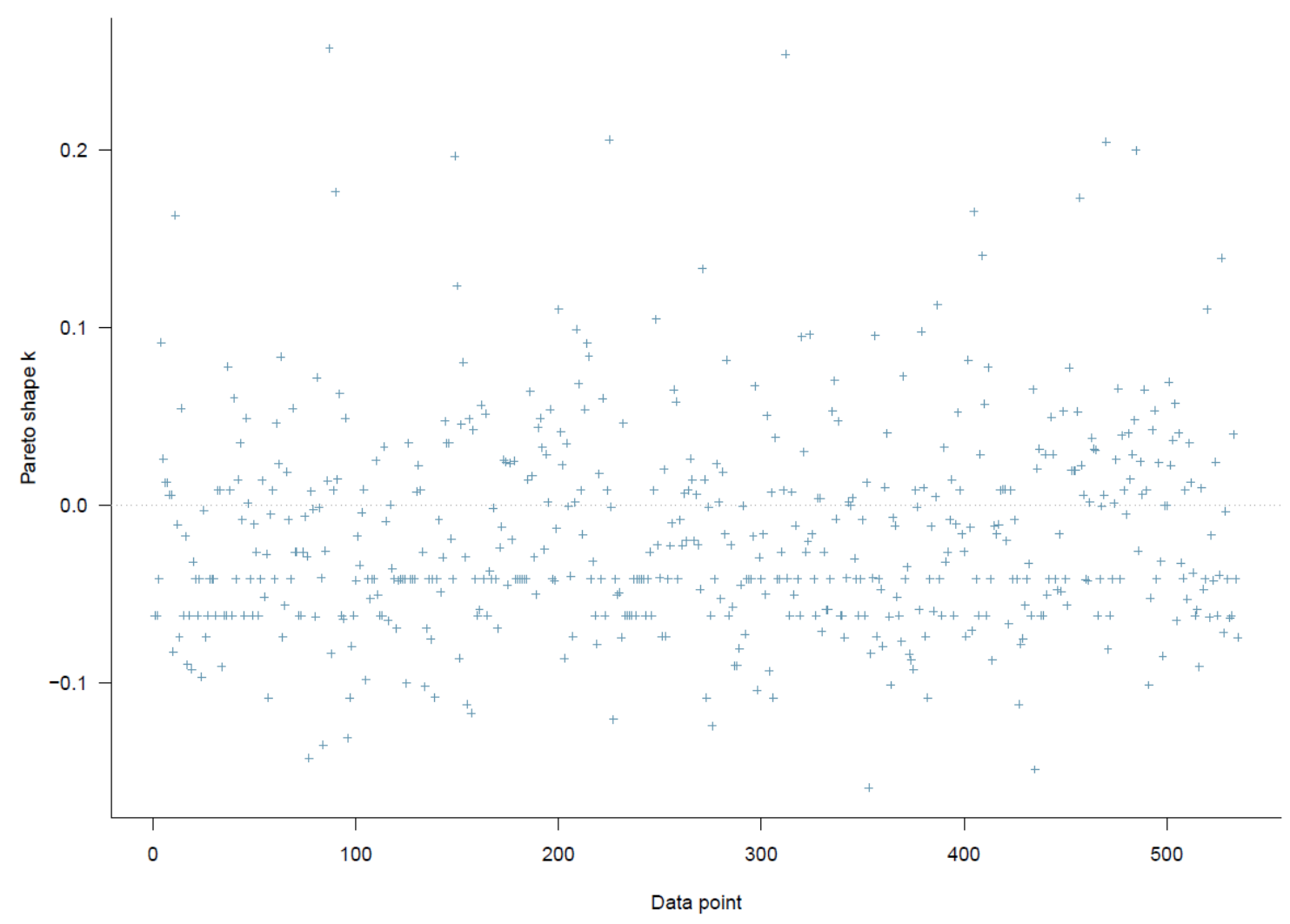

Figure 1: Model 1's PSIS-LOO diagnosis with priors as norm $(1,0.5)$

Next, it is necessary to verify the convergence of the model using two diagnostic values: effective sample size (n_eff) and Gelman shrink factor (Rhat). The n_eff value indicates the number of iterative samples that are not autocorrelated during the stochastic simulation process. Generally, it is accepted that if the $n$ _eff value is greater than 1000, the Markov chains are convergent, and the effective samples are enough for accurate inference. In terms of the Rhat value, if the value is above 1, it implies that the chains have not converged, so inference should not be made with the current iterative samples. On the contrary, if the value is equal to 1, it is a good convergence signal. As the parameters' $\mathrm{n}$ _eff values are all larger than 9000 and Rhat values are equal to 1, Model 1 seems to have good convergence, even when priors used are different (Table 2).

Table 2:

\begin{tabular}{|c|c|c|c|c|c|c|c|c|}
\hline \multirow{2}{*}{ Parameters } & \multicolumn{4}{|c|}{$\begin{array}{c}\text { Informative priors } \\
\text { (belief on effect) }\end{array}$} & \multicolumn{4}{c|}{$\begin{array}{c}\text { Informative priors } \\
\text { (disbelief on effect) }\end{array}$} \\
\cline { 2 - 10 } & Mean & SD & n_eff & Rhat & Mean & SD & n_eff & Rhat \\
\hline Constant & 1.20 & 0.14 & 12522 & 1 & 1.22 & 0.14 & 12512 & 1 \\
\hline EnvironmentalDegradation & 0.35 & 0.05 & 10215 & 1 & 0.35 & 0.05 & 12151 & 1 \\
\hline EconomicGrowthLoss & 0.05 & 0.04 & 11215 & 1 & 0.05 & 0.04 & 11512 & 1 \\
\hline NatureRecreationLoss & 0.18 & 0.07 & 9212 & 1 & 0.18 & 0.07 & 10215 & 1 \\
\hline HealthLoss & -0.05 & 0.05 & 11215 & 1 & -0.05 & 0.05 & 12562 & 1 \\
\hline KnowledgeLoss & 0.13 & 0.04 & 12841 & 1 & 0.13 & 0.04 & 12354 & 1 \\
\hline
\end{tabular}


The convergence is validated again using the trace, Gelman, and autocorrelation plots. Figure 2 demonstrates the trace plots of Model 1, which indicate that the Markov chains are all convergent. Two signals can be used to diagnose convergence. First, the Markov chains are good mixing, illustrated by the rapid zig-zag motion of each line. Second, the Markov chains are stationary, or the chains only stay within the posterior distribution. In Figure 2, all iterations before the $2000^{\text {th }}$ order are removed since warmup iterations are not used for inference.
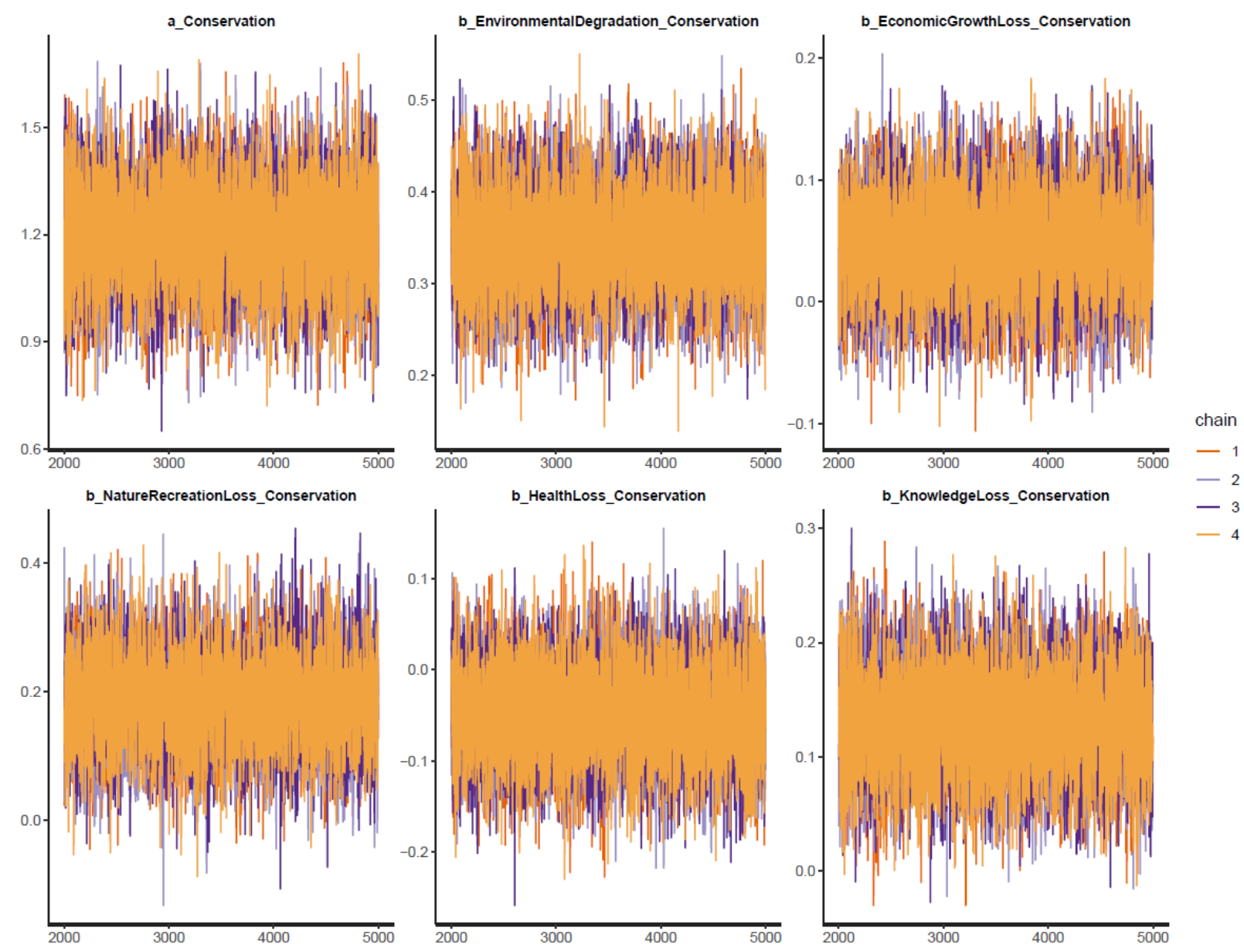

Figure 2: Model 1's trace plots with priors as norm $(1,0.5)$

A Gelman plot is the visualization of the Gelman shrink factor (y-axis) corresponding to the sequential order of iterations (x-axis). As shown in Figure 3, the Gelman shrink factor values drop rapidly to 1 before the warmup period ends (before the $2000^{\text {th }}$ iteration). This signals a good convergence of Model 1. 

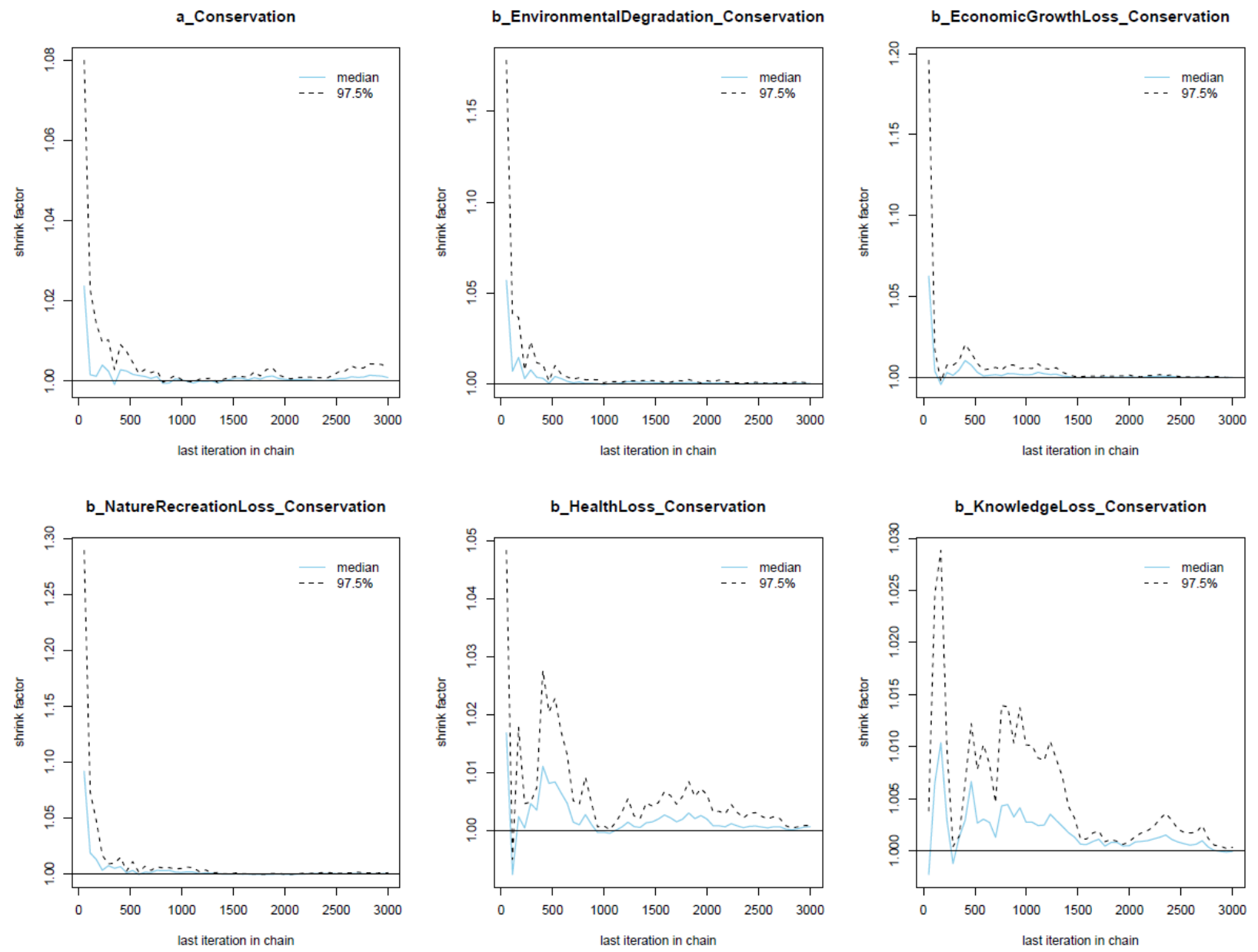

Figure 3: Model 1's Gelman plots with priors as norm $(1,0.5)$

The last diagnostic plot of model convergence is the autocorrelation plot. For a model's Markov chains to converge, the stochastic process has to present the memoryless property. In other words, the simulated samples are independent of previously simulated samples. The autocorrelation plots display a rapid decline of autocorrelation level to 0 after a finite lag, validating that the model's Markov chains are convergent. 

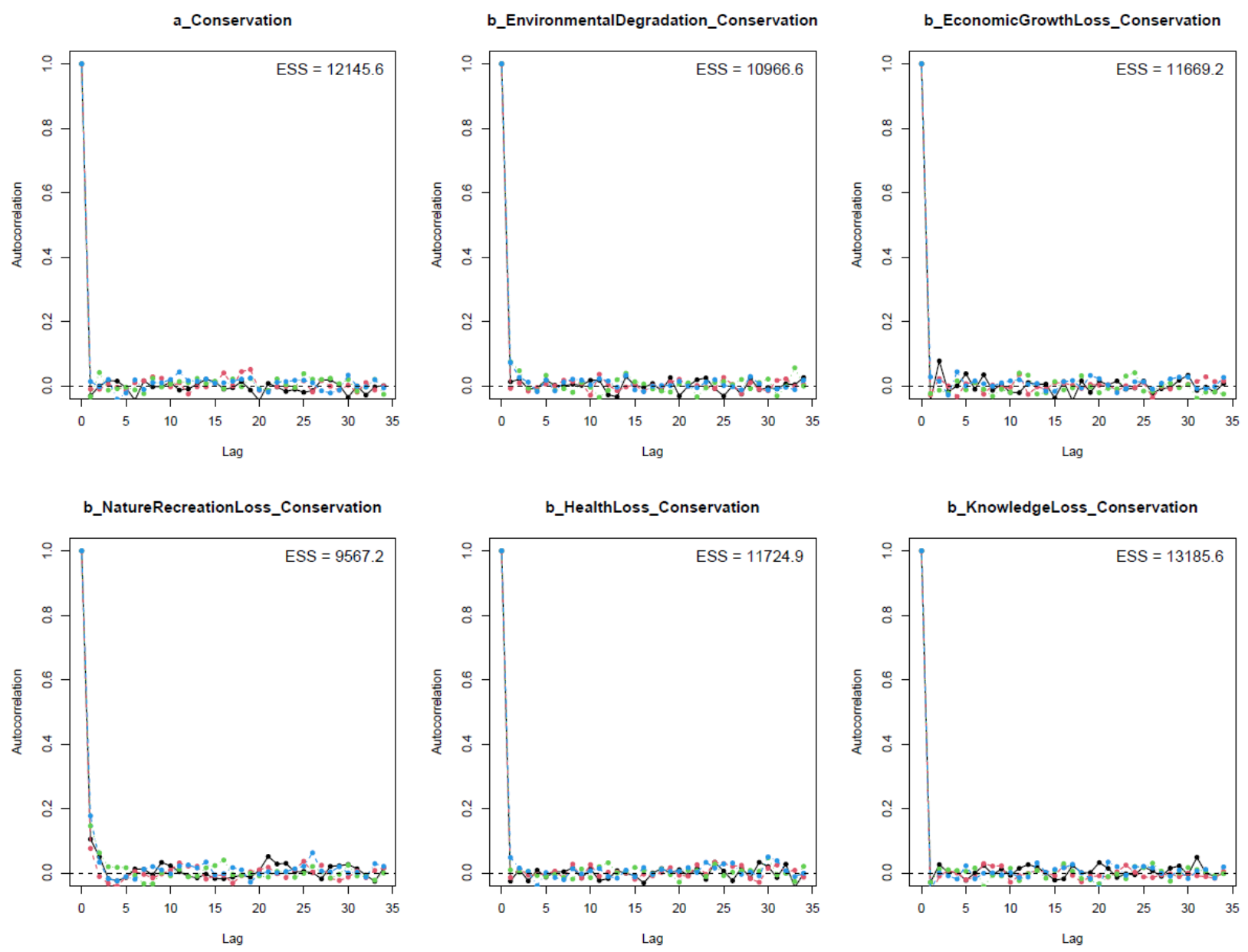

Figure 4: Model 1's autocorrelation plots with priors as norm $(1,0.5)$

The simulated posteriors employing priors as norm $(1,0.5)$ show that four out of five biodiversity loss perceptions are positively associated with the conservation-related attitude, namely: environmental degradation ( $\mu_{\text {EnvironmentalDegradation }}=0.35, \sigma_{\text {EnvironmentalDegradation }}=0.05$ ), loss of economic growth $\left(\mu_{\text {EconomicGrowthLoss }}=0.05, \sigma_{\text {EconomicGrowthLoss }}=0.04\right)$, loss of naturebased recreation opportunity $\left(\mu_{\text {NatureRecreationLoss }}=0.18, \sigma_{\text {NatureRecreationLoss }}=0.07\right)$, and loss of knowledge ( $\left.\mu_{\text {KnowledgeLoss }}=0.13, \sigma_{\text {KnowledgeLoss }}=0.04\right)$. Interestingly, perceiving loss of health as a consequence of biodiversity loss has an opposite effect on conservation-related attitude $\left(\mu_{\text {HealthLoss }}=-0.05, \sigma_{\text {HealthLoss }}=0.05\right)$.

The parameters' posterior distributions are shown in Figure 5, along with their Highest Posterior Distribution Interval (HPDI) at 90\%. Apparently, all the credible intervals of EnvironmentalDegradation, EconomicGrowthLoss, NatureRecreationLoss, and KnowledgeLoss fall entirely on the positive side of the $\mathrm{x}$-axis, suggesting that the positive associations between these variables and outcome variable (Conservation) are highly reliable. Regarding HealthLoss's posterior distribution, the majority of its HPDI is located on the negative side but not entirely, and its standard deviation (SD) is equal to the absolute value of the mean, so HealthLoss's negative effect on conservation-related attitude can be deemed moderately reliable. Even "prior-tweaking" 
is performed using the priors representing our disbelief on the associations between biodiversity loss perceptions and conservation-related attitude, the change is negligible, which indicates the model's robustness (see Table 2).
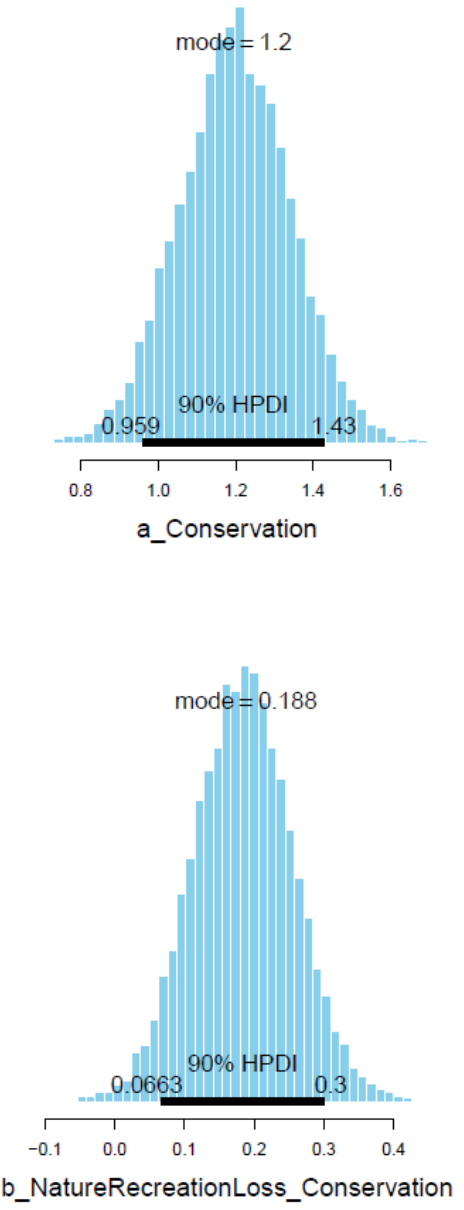
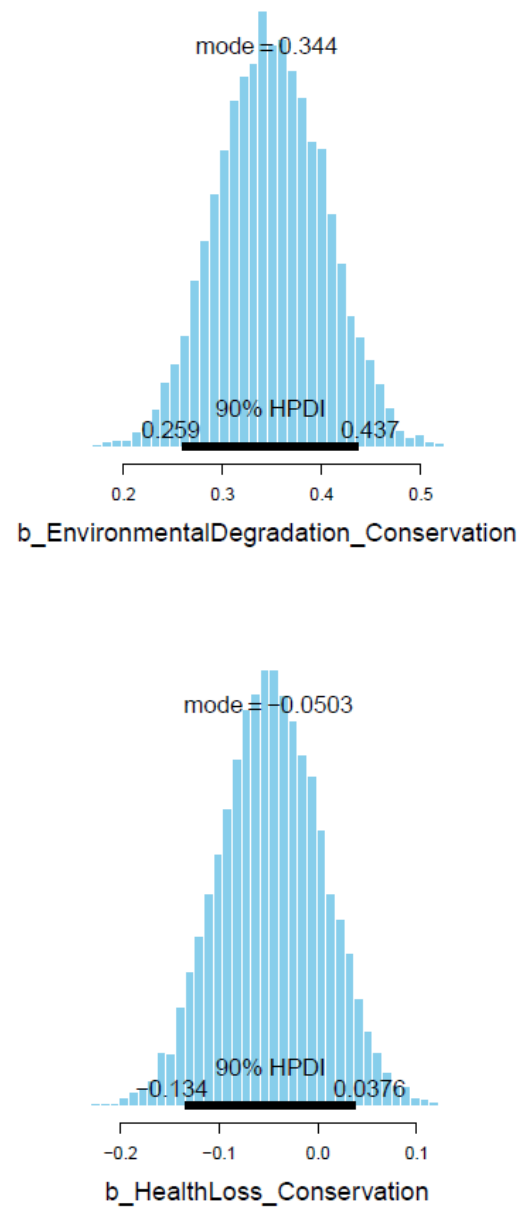
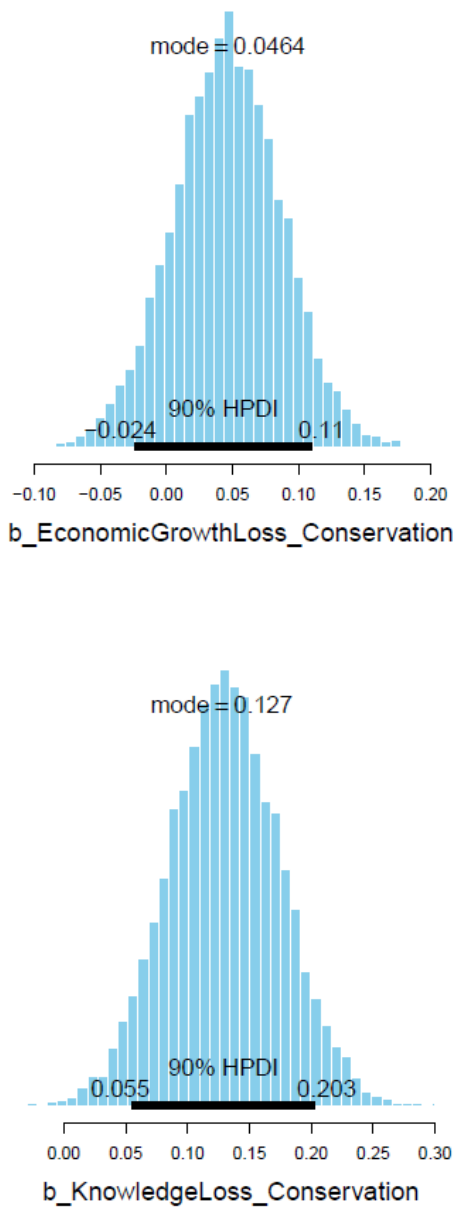

Figure 5: Model 1's posterior distributions with priors as norm $(1,0.5)$

\section{Models $2 \mathrm{a}$ and $2 \mathrm{~b}$}

Models $2 \mathrm{a}$ and $2 \mathrm{~b}$ were examined to check whether urban residents' conservation-related attitude has positive impacts on their willingness to pay for the entrance fee and conservation when visiting protected areas in the future. We applied the same fitting and validating procedures of Models $2 \mathrm{a}$ and $2 \mathrm{~b}$ with Model 1 . The visual PSIS-LOO diagnoses of Models $2 \mathrm{a}$ and $2 \mathrm{~b}$ are displayed in Figures 6-A and 6-B, respectively. $k$-values in both Figures are below the 0.5 thresholds, so Models $2 \mathrm{a}$ and $2 \mathrm{~b}$ have a good fit with the data.

Convergence diagnostic values (n_eff and Rhat) of both models indicate that the models' Markov chains are convergent. The trace, Gelman, and autocorrelation plots also confirm the model convergence. Figures A1, A2, and A3 are the trace, Gelman, and autocorrelation plots of Model 2a, respectively, while those of Model $2 \mathrm{~b}$ are presented in Figures A4, A5, and A6. 
Table 3:

\begin{tabular}{|c|c|c|c|c|c|c|c|c|}
\hline \multicolumn{9}{|c|}{ Model 2a: WillingEntraceFee $\sim$ Conservation } \\
\hline \multirow[t]{2}{*}{ Parameters } & \multicolumn{4}{|c|}{$\begin{array}{l}\text { Informative priors } \\
\text { (belief on effect) }\end{array}$} & \multicolumn{4}{|c|}{$\begin{array}{l}\text { Informative priors } \\
\text { (disbelief on effect) }\end{array}$} \\
\hline & Mean & SD & n_eff & Rhat & Mean & SD & n_eff & Rhat \\
\hline Constant & 1.23 & 0.80 & 2648 & 1 & 2.04 & 0.89 & 2542 & 1 \\
\hline Conservation & 0.81 & 0.26 & 2643 & 1 & 0.53 & 0.28 & 2342 & 1 \\
\hline \multicolumn{9}{|c|}{ Model 2b: WillingDonation Conservation } \\
\hline Parameters & \multicolumn{4}{|c|}{$\begin{array}{l}\text { Informative priors } \\
\text { (belief on effect) }\end{array}$} & \multicolumn{4}{|c|}{$\begin{array}{l}\text { Informative priors } \\
\text { (disbelief on effect) }\end{array}$} \\
\hline Constant & 0.28 & 0.63 & 2698 & 1 & 0.76 & 0.66 & 2324 & 1 \\
\hline Conservation & 0.86 & 0.21 & 2517 & 1 & 0.70 & 0.21 & 2321 & 1 \\
\hline
\end{tabular}
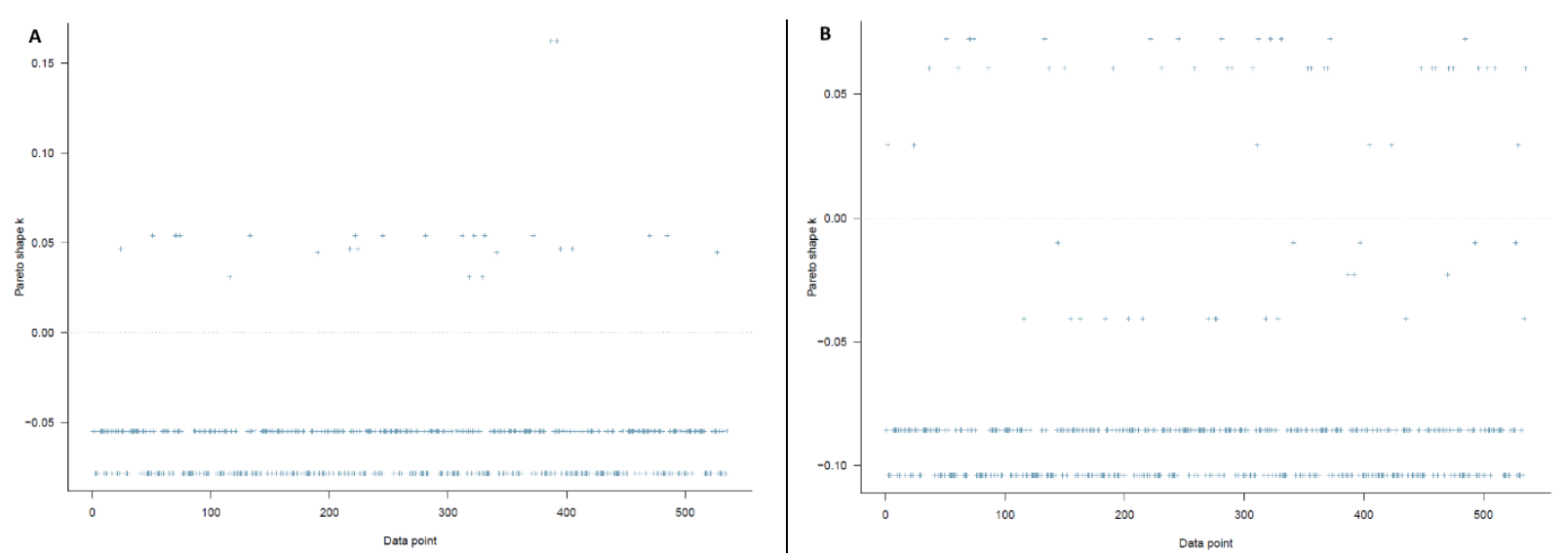

Figure 6: PSIS-LOO diagnosis for A) Model 2a and B) Model 2b with priors as norm $(1,0.5)$

As can be seen from Table 3, people with a higher agreement level with conservation as a preventive measure of biodiversity loss are more willing to pay for entrance fee $\left(\mu_{\text {Conservation_WillingEntranceFee }}=0.81, \sigma_{\text {Conservation_WillingEntranceFee }}=0.26\right)$ and conservation $\left(\mu_{\text {Conservation_WillingDonation }}=0.86, \sigma_{\text {Conservation_WillingDonation }}=0.21\right)$. The posterior distributions of the parameters representing the association between conservation-related attitude and willingness to pay for entrance fee and conservation are displayed in Figures 7-A and 7-B, respectively. The distributions clearly lie on the positive side of the $\mathrm{X}$-axis (separated by the red vertical line), stipulating highly reliable positive associations. When estimating Models $2 \mathrm{a}$ and $2 \mathrm{~b}$ employing priors as norm $(0,0.5)$, the magnitude of the posterior distribution declines, its reliability is still high. Evidently, their mean values are much greater than the standard deviation values. 

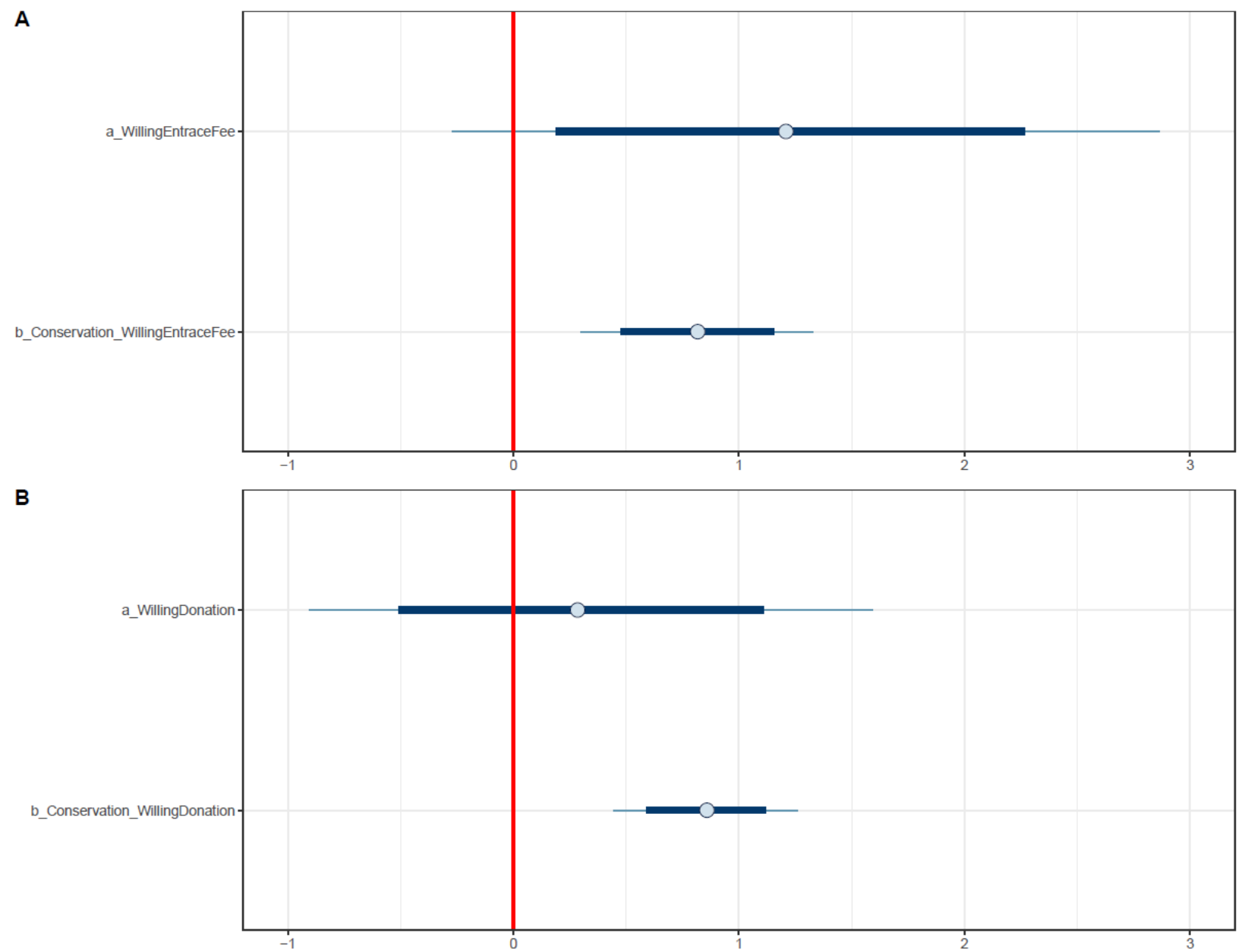

Figure 7: Interval plots of posterior distributions for A) Model 2a and B) Model 2b

\section{Model 3}

Fitting Models $3 \mathrm{a}$ and $3 \mathrm{~b}$, we aimed to examine the predictions of conservation-related attitude and biodiversity perceptions against the willingness to pay for the entrance fee and conservation in protected areas. The fitting and validating procedures are also similar to those employed with Model 1. First of all, PSIS-LOO diagnosis was conducted with both models. The visualizations of $k$-values (all $k$-values are lower than 0.5) in Figures 8-A and 8-B show that Models 3a and 3b are neither underfit nor overfit with the data. 

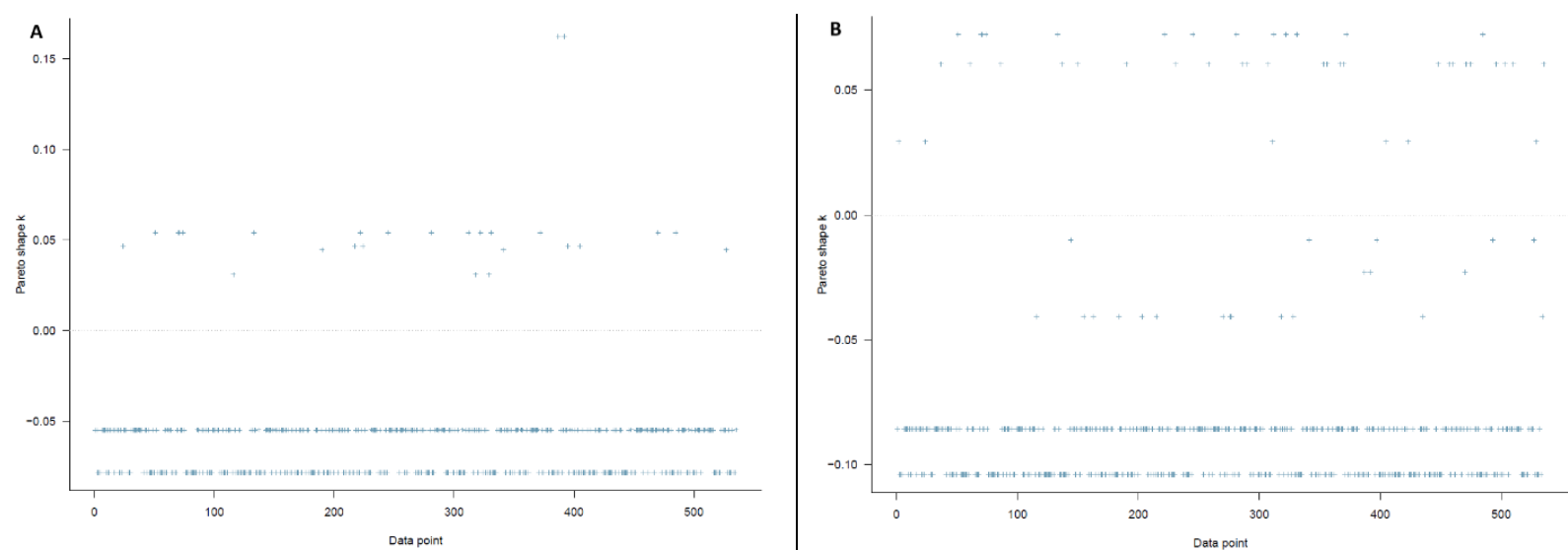

Figure 8: PSIS-LOO diagnosis for A) Model 3a and B) Model 3b with priors as norm $(1,0.5)$

The n_eff and Rhat values presented in Table 4 confirm the convergence of Models $3 \mathrm{a}$ and $3 \mathrm{~b}$ (n_eff > 8000 and Rhat $=1$ ). The visual diagnoses by trace, Gelman, and autocorrelation plots also verify the convergence. Figures A7, A8, and A9 demonstrate Model 3a's trace, Gelman, and autocorrelation plots, while Figures A10, A11, and A12 are Model 3b's trace, Gelman, and autocorrelation plots, respectively. Interpretation of the plots can be viewed in sub-section 3.1.

Table 4:

\begin{tabular}{|c|c|c|c|c|c|c|c|c|}
\hline \multicolumn{9}{|c|}{$\begin{array}{l}\text { Model 3a: WillingEntraceFee } \sim \text { Conservation + EnvironmentalDegradation+ } \\
\text { EconomicGrowthLoss+ NatureRecreationLoss + HealthLoss + KnowledgeLoss }\end{array}$} \\
\hline \multirow{2}{*}{ Parameters } & \multicolumn{4}{|c|}{$\begin{array}{l}\text { Informative priors } \\
\text { (belief on effect) }\end{array}$} & \multicolumn{4}{|c|}{$\begin{array}{l}\text { Informative priors } \\
\text { (disbelief on effect) }\end{array}$} \\
\hline & Mean & SD & n_eff & Rhat & Mean & SD & n_eff & Rhat \\
\hline Constant & 0.06 & 0.96 & 9412 & 1 & 1.49 & 1.14 & 8421 & 1 \\
\hline Conservation & 0.51 & 0.31 & 9778 & 1 & 0.39 & 0.31 & 10332 & 1 \\
\hline EnvironmentalDegradation & -0.09 & 0.35 & 9321 & 1 & -0.15 & 0.35 & 9221 & 1 \\
\hline EconomicGrowthLoss & 0.15 & 0.33 & 10654 & 1 & 0.03 & 0.33 & 10963 & 1 \\
\hline NatureRecreationLoss & 0.22 & 0.40 & 10596 & 1 & 0.13 & 0.39 & 9654 & 1 \\
\hline HealthLoss & -0.09 & 0.37 & 9632 & 1 & -0.18 & 0.37 & 9231 & 1 \\
\hline KnowledgeLoss & 0.61 & 0.32 & 10212 & 1 & 0.55 & 0.31 & 10321 & 1 \\
\hline \multicolumn{9}{|c|}{$\begin{array}{l}\text { Model 3b: WillingDonation } ~ \text { Conservation + EnvironmentalDegradation+ } \\
\text { EconomicGrowthLoss + NatureRecreationLoss + HealthLoss+ KnowledgeLoss }\end{array}$} \\
\hline Parameters & \multicolumn{4}{|c|}{$\begin{array}{l}\text { Informative priors } \\
\text { (belief on effect) }\end{array}$} & \multicolumn{4}{|c|}{$\begin{array}{l}\text { Informative priors } \\
\text { (disbelief on effect) }\end{array}$} \\
\hline Constant & -0.47 & 0.63 & 10512 & 1 & 0.37 & 0.84 & 10393 & 1 \\
\hline Conservation & 0.64 & 0.21 & 10517 & 1 & 0.57 & 0.25 & 10417 & 1 \\
\hline EnvironmentalDegradation & 0.18 & 0.30 & 9232 & 1 & 0.14 & 0.30 & 10963 & 1 \\
\hline EconomicGrowthLoss & -0.02 & 0.27 & 10351 & 1 & -0.09 & 0.26 & 11736 & 1 \\
\hline NatureRecreationLoss & -0.09 & 0.35 & 11542 & 1 & -0.13 & 0.35 & 9551 & 1 \\
\hline
\end{tabular}




\begin{tabular}{|c|c|c|c|c|c|c|c|c|}
\hline HealthLoss & 0.07 & 0.31 & 8021 & 1 & 0.02 & 0.31 & 10789 & 1 \\
\hline KnowledgeLoss & 0.35 & 0.27 & 10123 & 1 & 0.32 & 0.27 & 10545 & 1 \\
\hline
\end{tabular}

The simulated posterior results of Models $3 \mathrm{a}$ and $3 \mathrm{~b}$ show that the positive associations between conservation-related attitude and willingness to pay for entrance fee and conservation remain robust with Models 2a's and 2b's results. Most of the biodiversity loss perceptions' effects on both willingness to pay for the entrance fee and conservation are negligible and unreliable. In particular, their standard deviation values are much higher than the means' absolute values. Only KnowledgeLoss has positive effects on the willingness to pay for the entrance fee $\left(\mu_{\text {KnowledgeLoss_WillingEntranceFee }}=0.61, \quad \sigma_{\text {KnowledgeLoss_WillingEntranceFee }}=0.32\right)$ and conservation $\left(\mu_{\text {KnowledgeLoss_WillingDonation }}=0.35, \sigma_{\text {KnowledgeLoss_WillingDonation }}=0.27\right)$.

The interval plots of Models 3a's and 3b's posterior distributions manifest that Conservation's and KnowledgeLoss's HPDIs at $90 \%$ are entirely located on the positive side, highlighting the high reliability of their effects on willingness to pay. The HPDI at $90 \%$ is illustrated by the thick part in the middle of an interval. After conducting the "prior-tweaking" technique, the parameters' magnitudes slightly change, but their tendencies are not. Hence, the simulated results are robust.
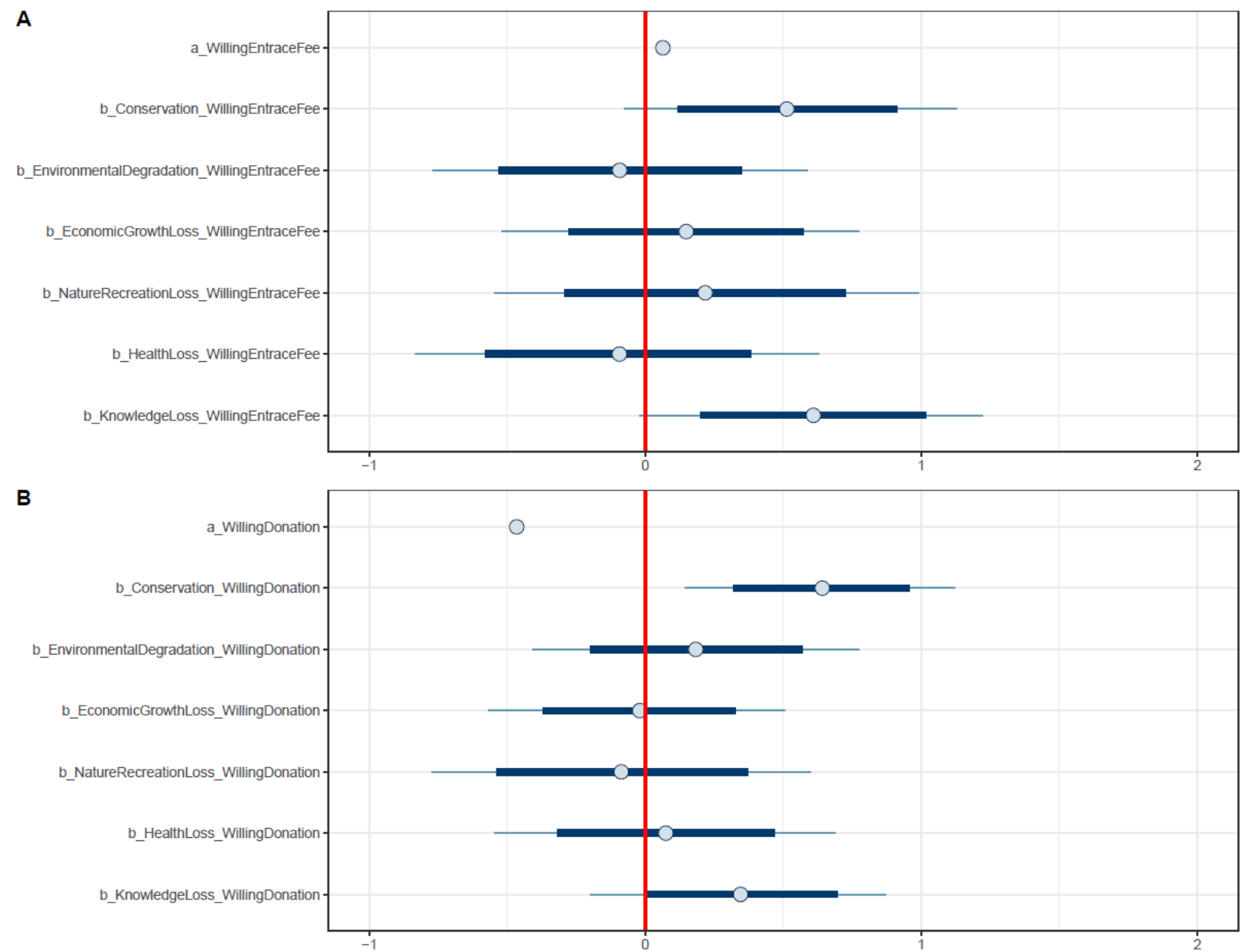
Figure 9: Interval plots of posterior distributions for A) Model 3a and B) Model 3b

Based on the results reported above, it is conclusive that biodiversity loss perceptions (EnvironmentalDegradation, EconomicGrowthLoss, NatureRecreationLoss, and KnowledgeLoss) have direct positive impacts on conservation-related attitude and indirect positive impacts on willingness to pay for entrance fee and conservation through affecting the conservation-related attitude. Perceiving the loss of knowledge as a consequence of biodiversity loss directly positively influences the conservation-related attitude and willingness to pay.

\section{Discussion}

The current study is one of the first studies examining how the urban residents' biodiversity loss perceptions associate with their conservation-related attitude and willingness to pay for the entrance fee and conservation in protected areas. The analysis was performed using the BMF on 535 urban inhabitants across Vietnam. Overall, there are three main findings: 1) most biodiversity loss perceptions (EnvironmentalDegradation, EconomicGrowthLoss, NatureRecreationLoss, and KnowledgeLoss) have direct positive impacts on conservation-related attitude and indirect impacts on willingness to pay; 2) perceiving loss of health as a consequence of biodiversity loss has negative influence the conservation-related attitude; 3) perceiving loss of knowledge as a consequence of biodiversity loss has a direct positive influence on conservation-related attitude and indirect positive influences on willingness to pay for entrance fee and conservation.

Evidence from this study suggests that there can be a novel way to improve protected areas financing actively. It is to build an eco-surplus culture among potential visitors to protected areas (to be more specific, urban inhabitants) by making them perceive the adversities of biodiversity loss.

For building an eco-surplus culture, improving the accessibility of urban inhabitants to information regarding biodiversity and biodiversity loss is vital. Without accessibility to biodiversity-related information, the urban inhabitants cannot know that biodiversity loss problems exist no matter how crucial and severe it is to their lives. Social marketing and demarketing programs, public awareness-raising campaigns, educational activities, and pro-environmental entertaining platforms (e.g., commercial games) are potential methods to create "touchpoints" between urban residents and the biodiversity-related information [51,56-58]. In addition, the effectiveness of biodiversityrelated information in changing perceptions, attitudes, and behaviors is required. As shown in this study's results, the message that can stimulate the subjective cost-benefit judgements of urban residents towards biodiversity loss might help build eco-surplus attitude (or supporting conservation as a preventive measure of biodiversity loss). Ryan, et al. [59] stipulate that the effectiveness of biodiversity-conservation marketing is still a nascent field with only 28 studies. For effectively building an eco-surplus culture, more knowledge regarding the effectiveness of the messages' content, narrative, and design in changing perceptions, attitudes, and behaviors is indispensable.

Building an eco-surplus culture is also a potential way to ease the funding allocation problems faced by the domestic government (e.g., widespread but insufficient budget allocation, lack of priority) and international organizations (e.g., large but site-specific funding) $[9,10]$. By financing 
social marketing and demarketing programs, public awareness-raising campaigns, educational activities, and pro-environmental entertaining platforms (e.g., commercial games), the government and international organization can increase the aggregate pool of money that the visitors are willing to pay at a regional scale, which indirectly generates finance for protected areas in the region. To elaborate, assuming that 5000 urbanites visit protected areas nearby the city every month. Before implementing pro-eco-culture campaigns and activities, $60 \%$ of them are willing to pay for the entrance fee and conservation initiatives, generating $\$ 60,000$ a month for protected areas in the region aggregately (each person pays $\$ 20$ ). It should be noted that $\$ 20$ per person is only an assuming number. After implementing pro-eco-culture campaigns and activities, $80 \%$ are willing to pay, generating $\$ 80,000$ ( $\$ 20,000$ surplus) for protected areas in the region. When the aggregate pool of money increases, all protected areas in the region will have an equal chance of benefiting through nature-based tourism [6,11]. The shifting demographics, rapid urbanization, exacerbating effects of climate change, increasing diffusion of media technologies, and changing psychological drivers will likely increase the demand for nature-based tourism swiftly in Asia-Pacific Region, especially developing countries like Vietnam [25]. In addition, the visitors with better informed knowledge about the effects of biodiversity and biodiversity loss might have more respect for nature and cause less impact to protected areas [60].

Implementing social marketing and demarketing programs, public awareness-raising campaigns, educational activities, and pro-environmental entertaining platform design (e.g., commercial games) does not only help increase the aggregate pool of finance for protected areas in the region but also facilitates other conservation campaigns in urban areas, like tackling illegal wildlife trade. Evidently, perceiving the consequences of biodiversity loss is found to influence the support towards prohibition of illegal wildlife consumption positively and negatively influence bushmeat consumption frequency among urban inhabitants [30]. Given the mentioned merits, we strongly recommend policymakers, international organizations, and conservation activists conduct targeted social marketing and demarketing programs, public awareness-raising campaigns, educational activities, and pro-environmental entertaining platform design (e.g., commercial games) to build an eco-surplus culture among urban residents for biodiversity conservation. Furthermore, enhancing the effectiveness of these campaigns and problems are promising research directions for scientists to contribute evidence-based insights.

The effects of biodiversity loss perceptions on paying willingness validate our assumptions about the role of the subjective cost-benefit evaluation process in accepting or rejecting information of the individuals. However, most of the effects of biodiversity loss perceptions on willingness to pay are indirect (except for the perceived loss of knowledge) and mediated by the attitude towards conservation, showing that the information evaluation process is sequential. In other words, it takes steps for a person to process information and eventually arrive at the ideations and behaviors that are beneficial for the environment or pro-environmental attitudes and behaviors. The impact of perceived knowledge loss is relatively special because it influences the willingness to pay both directly and indirectly through support for conservation. It is unclear why the effect of perceived knowledge is more direct than others, so investigating the link between perceived knowledge loss and support for conservation in general and willingness to pay in particular is a potential direction for later research. Regardless of the causes, the importance of knowledge about nature should be 
concentrated in public awareness-raising campaigns, social marketing and demarketing programs, and educational activities.

Regarding the negative effect of perceived health loss resulting from biodiversity loss on conservation-related attitude, it is paradoxical with other biodiversity loss perceptions' effects. Following the Mindsponge thinking, which assumes that people try to maximize their perceived benefits and reduce perceived cost, might help explain this finding [34]. In particular, urban residents who perceive health loss as a consequence of biodiversity loss are sensitive to healthrelated issues. In Vietnam, many perceived "nutritional" and "healthy" traditional medicines are made from wildlife products, such as pangolin scales, tiger bones, bear bile, etc. [61-63]. The term "conservation" might be perceived as a tool for protecting "a subset of biodiversity that includes charismatic species and those on threatened species lists" [64], so people sensitive to their health issues might be less likely to support conservation.

Moreover, the complex and sometimes ambiguous definition of biodiversity might also contribute to this contradiction. Apart from goods in an ecosystem, Vietnamese urban people also perceive "biodiversity" as an ecosystem itself [65]. People with this perception might consider the lack of fresh air (e.g., due to deforestation) as a health problem generated by biodiversity loss. It should be noted that the explanation here is speculative, so further studies are needed for validation. One potential approach is to look at how the subtle differences in people's perceptions (e.g., goods in an ecosystem, an ecosystem itself, a stage of equilibrium, its regulatory function, a final ecosystem service, etc.) about biodiversity might influence their environment-related thinking and behaviors [64-66].

Several limitations of this study are presented here for transparency [67]. The convenient sampling strategy due to the prolonged social distancing for COVID-19 containment may lead to selection bias, which reduces the generalization of the findings. By employing the Bayesian analysis, we could provide precise estimations based on the current dataset, which can be used to compare with studies analyzing random sampling data. Moreover, given the diverse residencies and background of participants (from cities across Vietnam), we believe our findings are still representative to some extent and should be used with caution. Another limitation is that there is no evidence that the willingness to pay before and after arriving at the protected areas will remain the same. Although there are possibilities that urban visitors' paying willingness decreases due to protected areas' characteristics and trip features, to some extent, the direct and indirect effects of biodiversity loss perceptions on willingness to pay are still reliable evidence for that improving awareness and knowledge among urban residents can lead to higher willingness to pay in protected areas.

\section{Acknowledgements}

We would like to send our gratitude to my family and friends for supporting me during the research, especially Prof. Vuong Quan Hoang (Phenikaa University) and Mr. Le Tam Tri (Phenikaa University).

Funding

Not applicable 
Conflicts of interest

This study is a part of the author's dissertation (M.-H.N).

Appendix
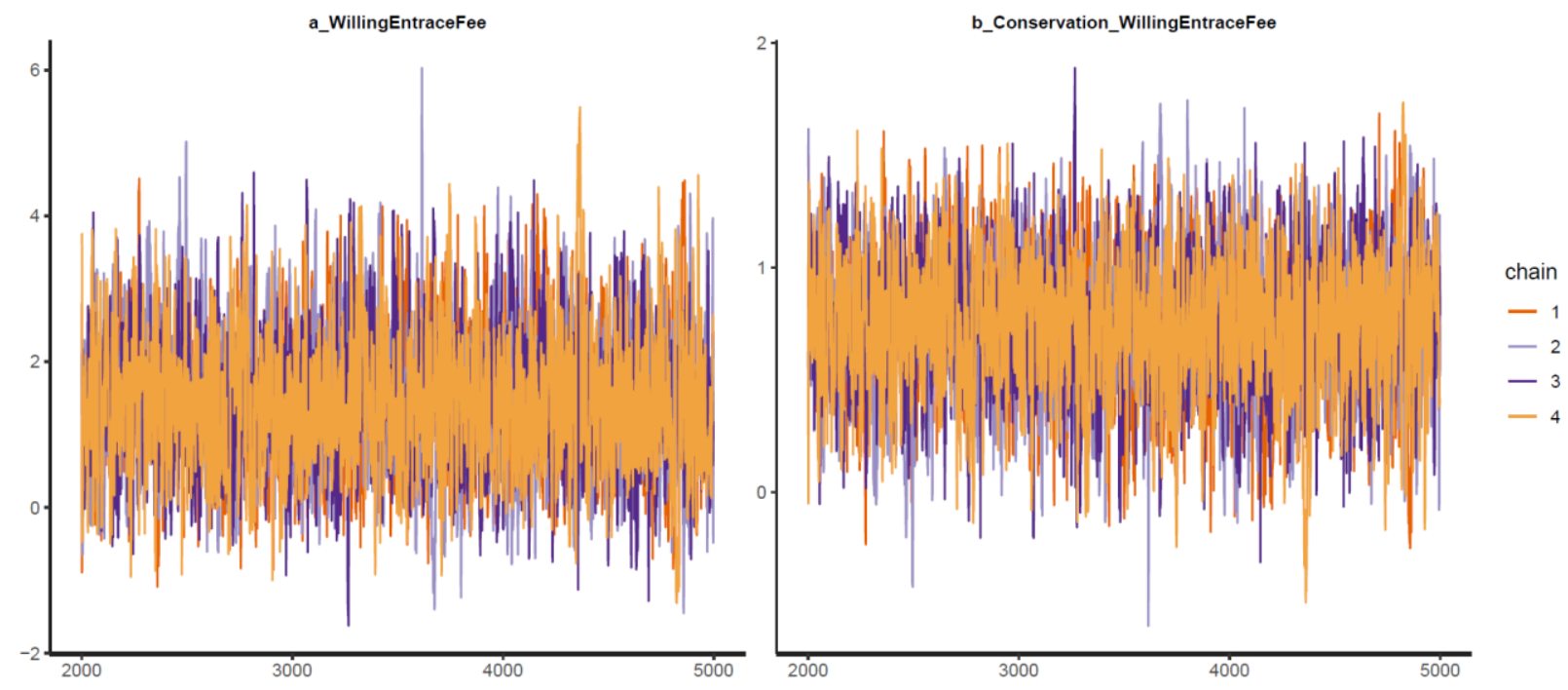

Figure A1: Model 2a's trace plots with priors as norm $(1,0.5)$
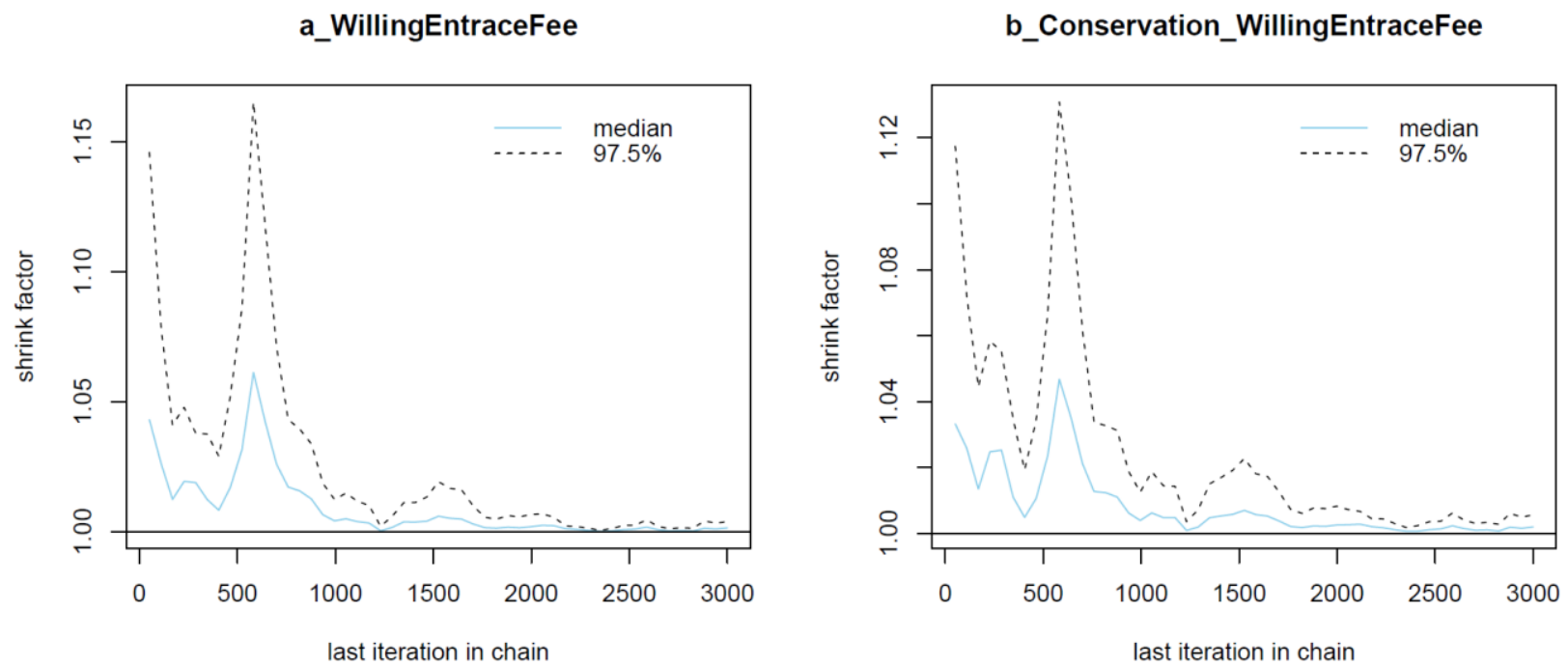

Figure A2: Model 2a's Gelman plots with priors as norm $(1,0.5)$ 
a_WillingEntraceFee

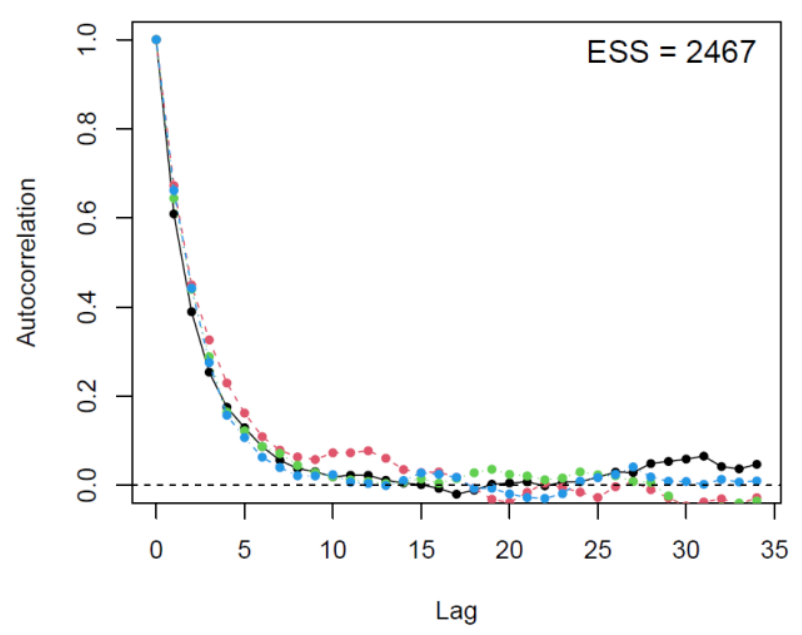

b_Conservation_WillingEntraceFee

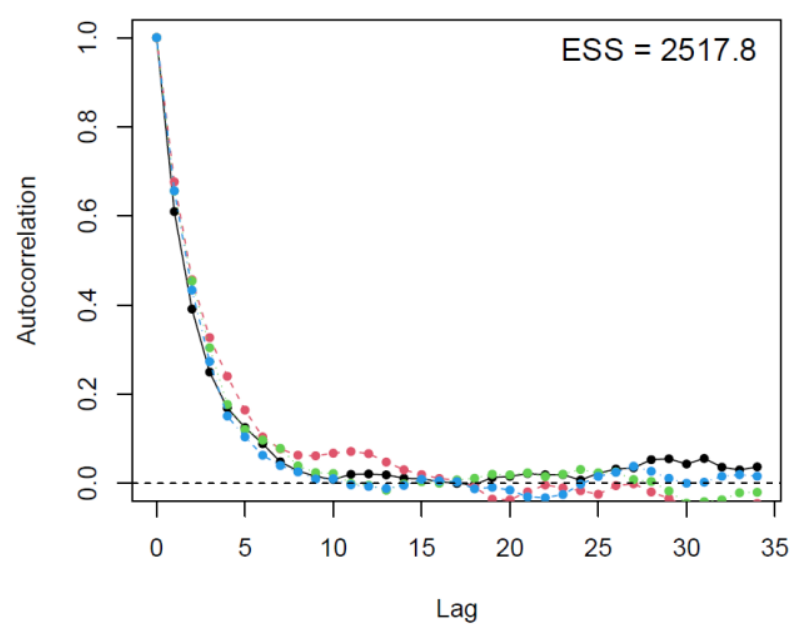

Figure A3: Model 2a's Gelman plots with priors as norm $(1,0.5)$
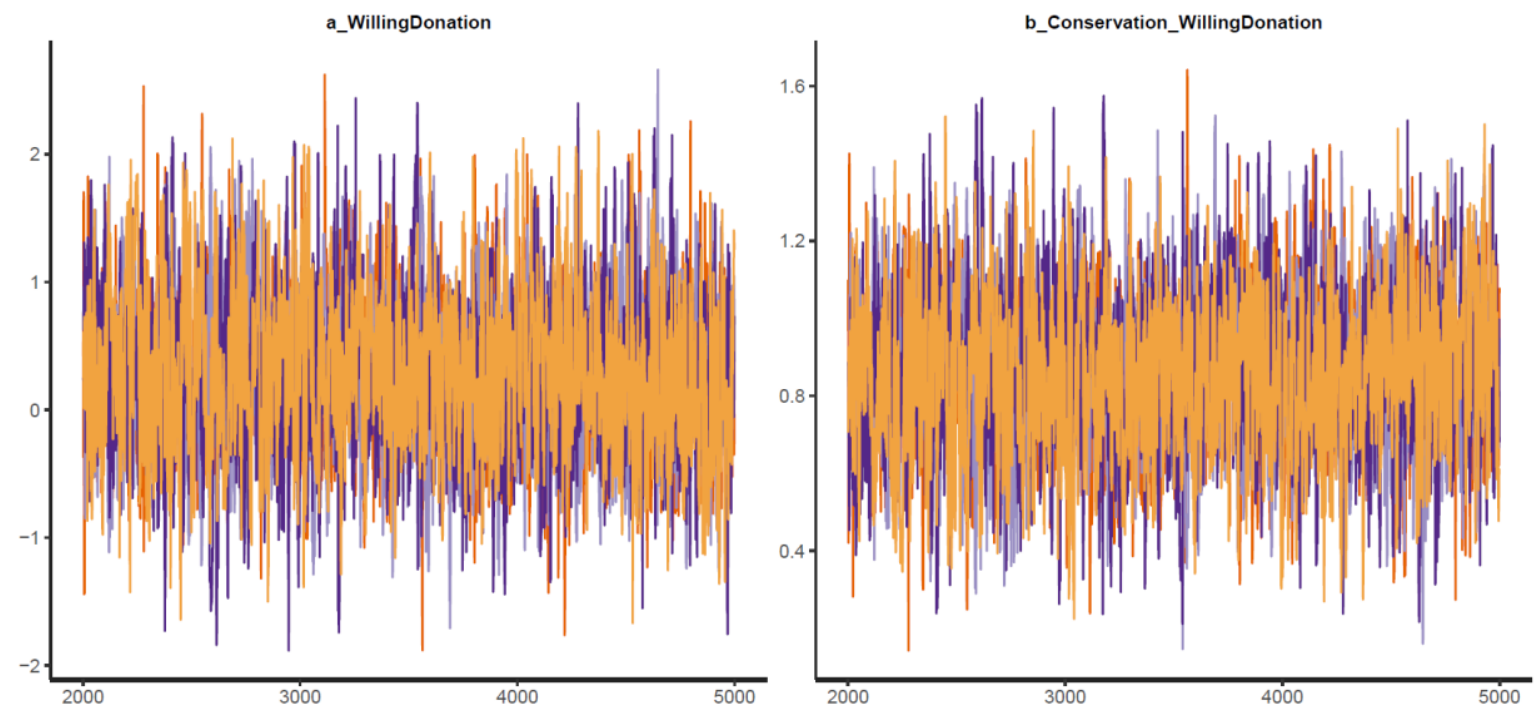

chain

$-1$

$-2$

$-3$

Figure A4: Model 2b's trace plots with priors as norm $(1,0.5)$ 

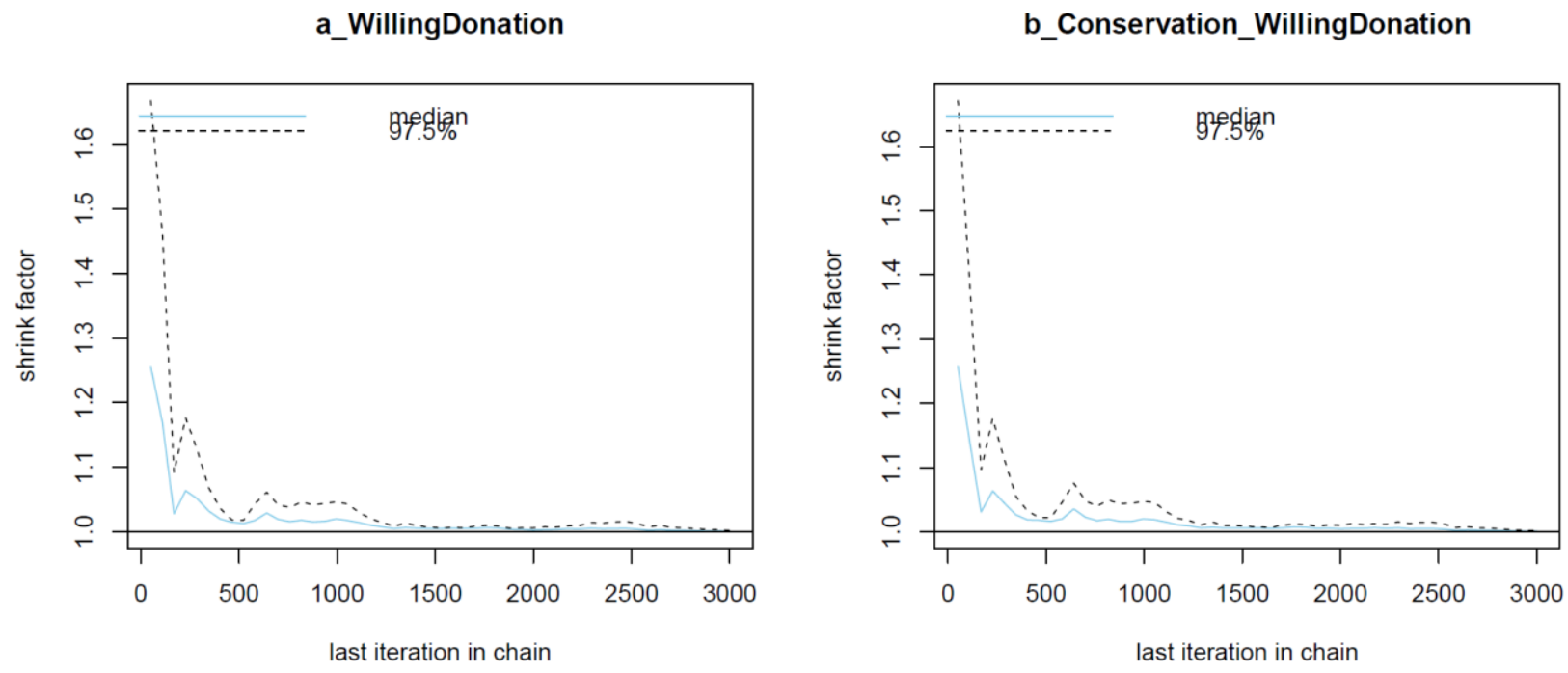

Figure A5: Model 2b's Gelman plots with priors as norm $(1,0.5)$
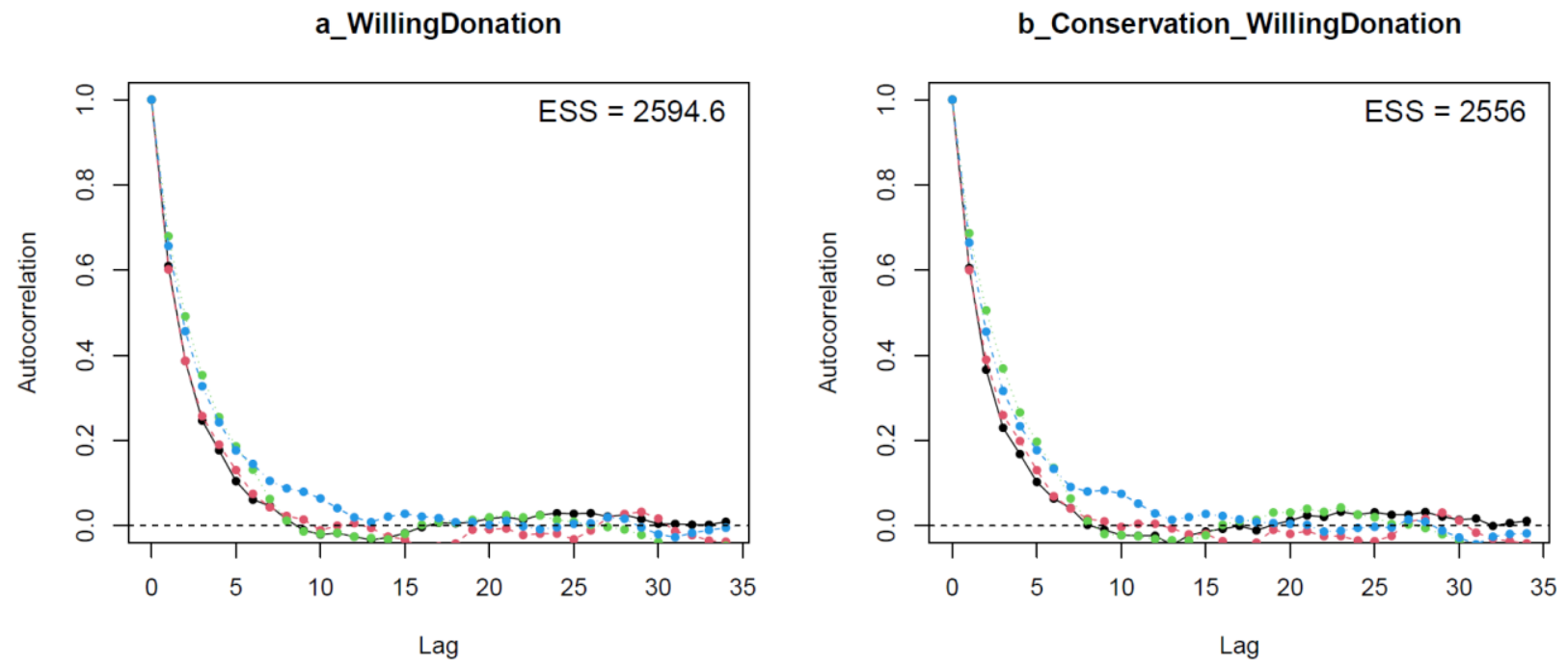

Figure A6: Model 2b's autocorrelation plots with priors as norm $(1,0.5)$ 

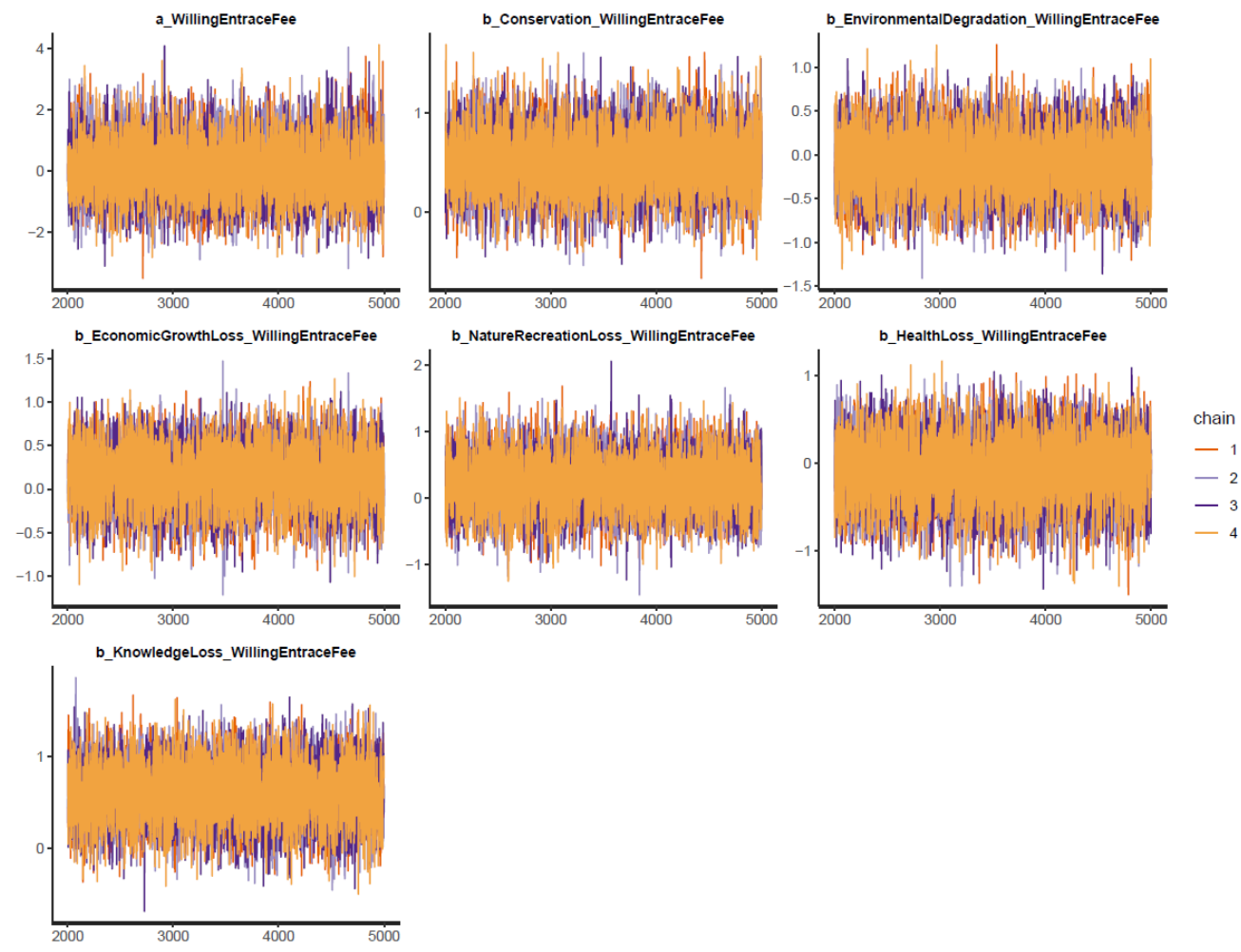

Figure A7: Model 3a's trace plots with priors as norm $(1,0.5)$
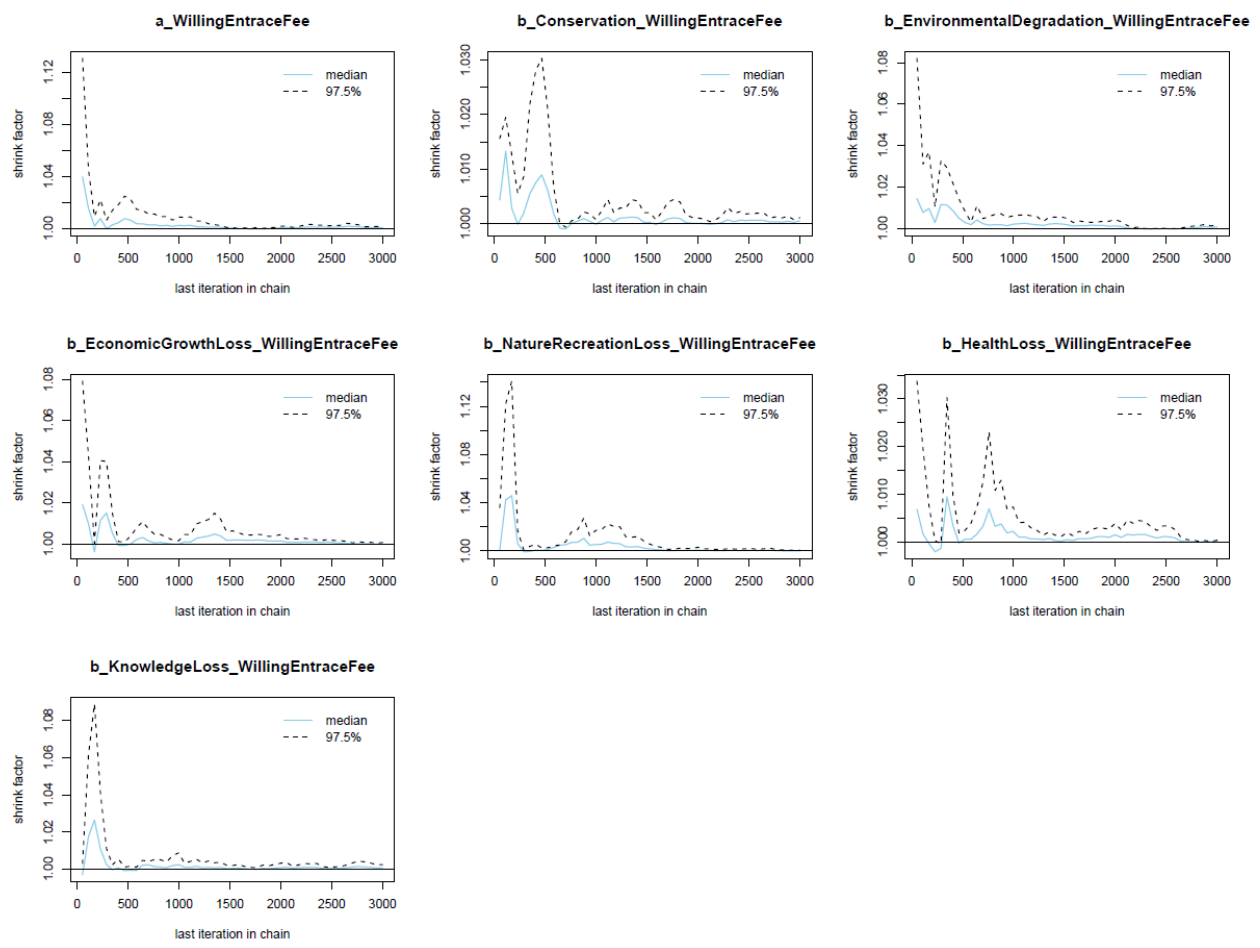

Figure A8: Model 3a's Gelman plots with priors as norm $(1,0.5)$ 

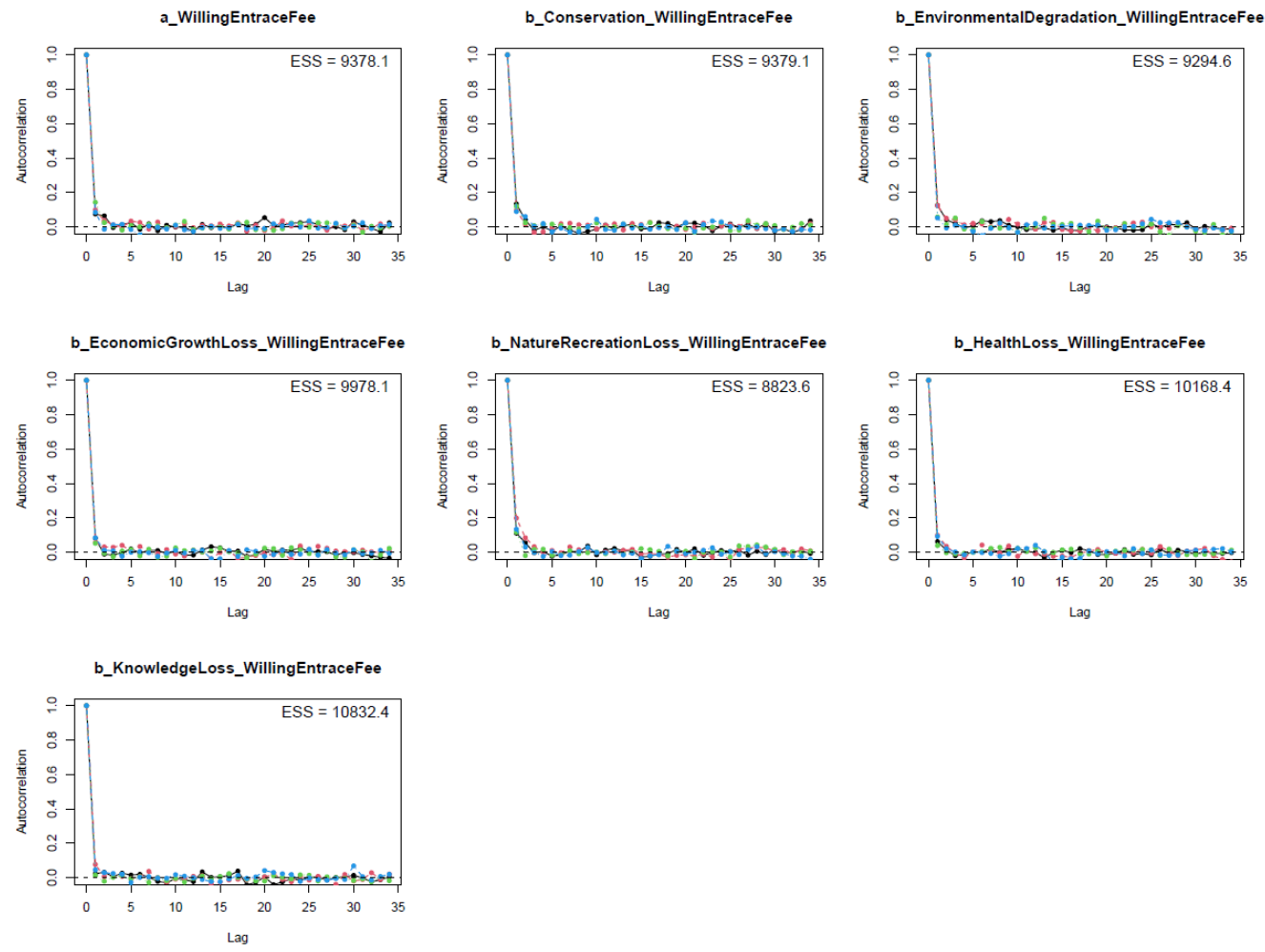

Figure A9: Model 3a's autocorrelation plots with priors as norm $(1,0.5)$
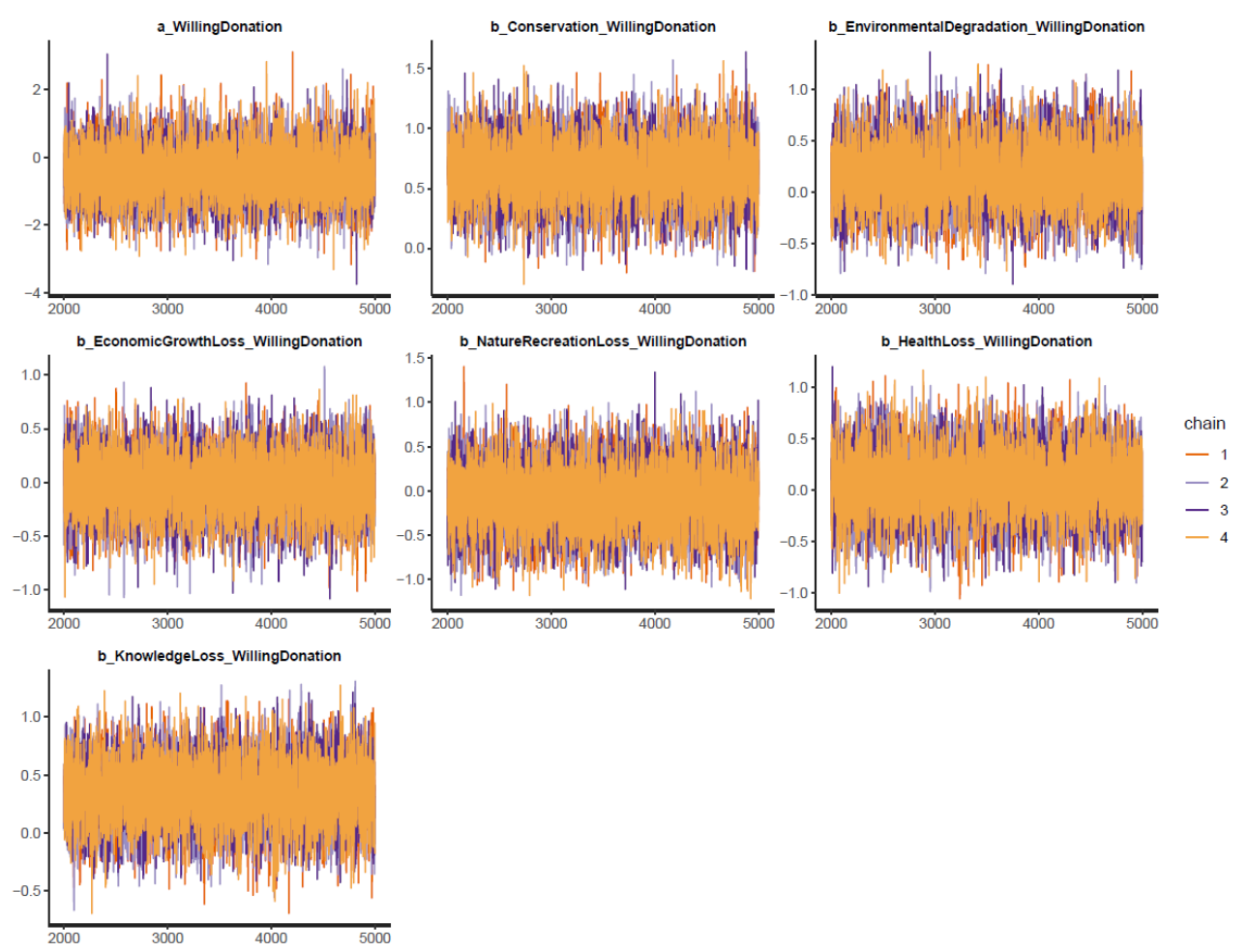

Figure A10: Model 3b's trace plots with priors as norm $(1,0.5)$ 

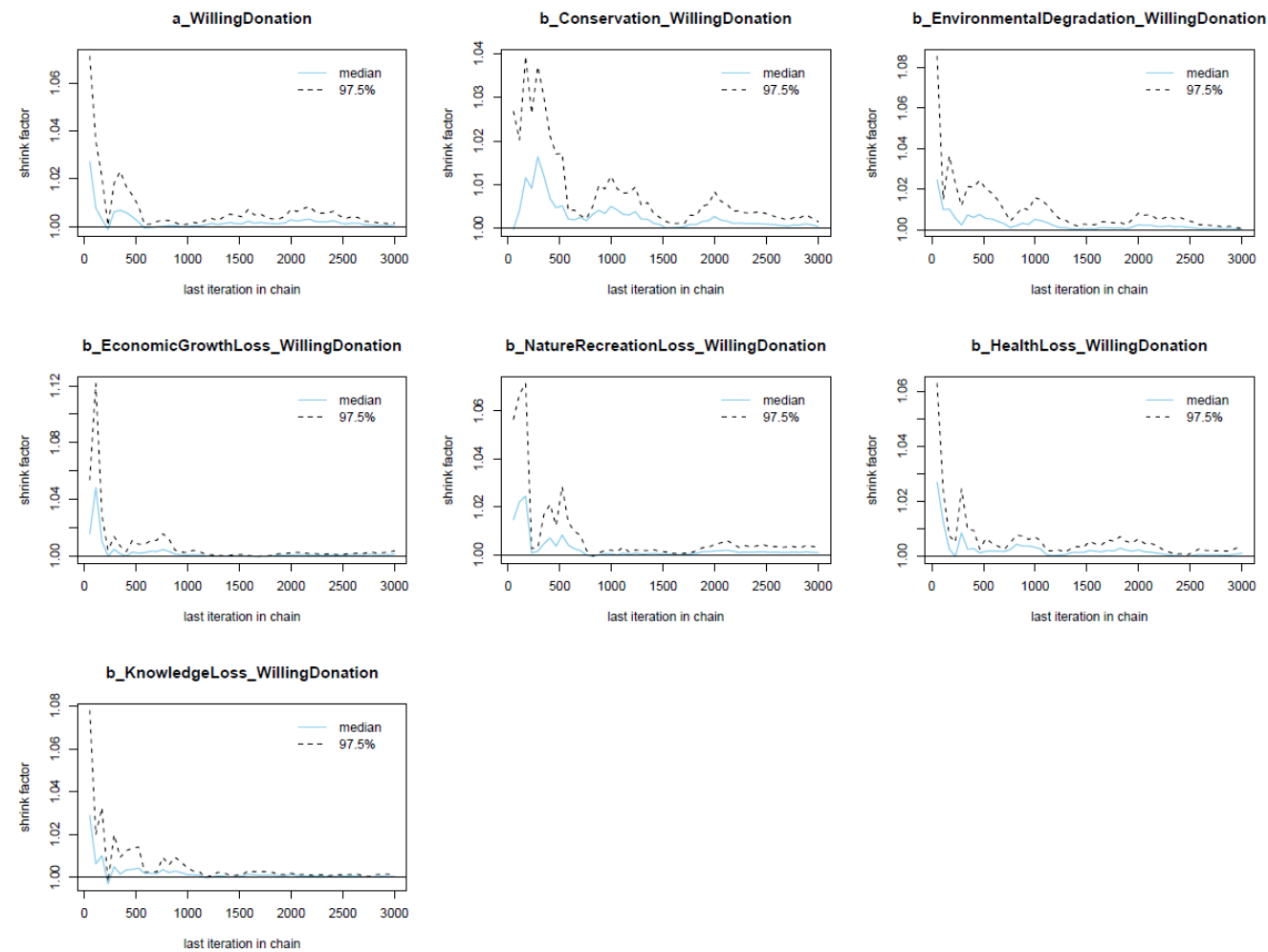

Figure A11: Model 3b's Gelman plots with priors as norm $(1,0.5)$
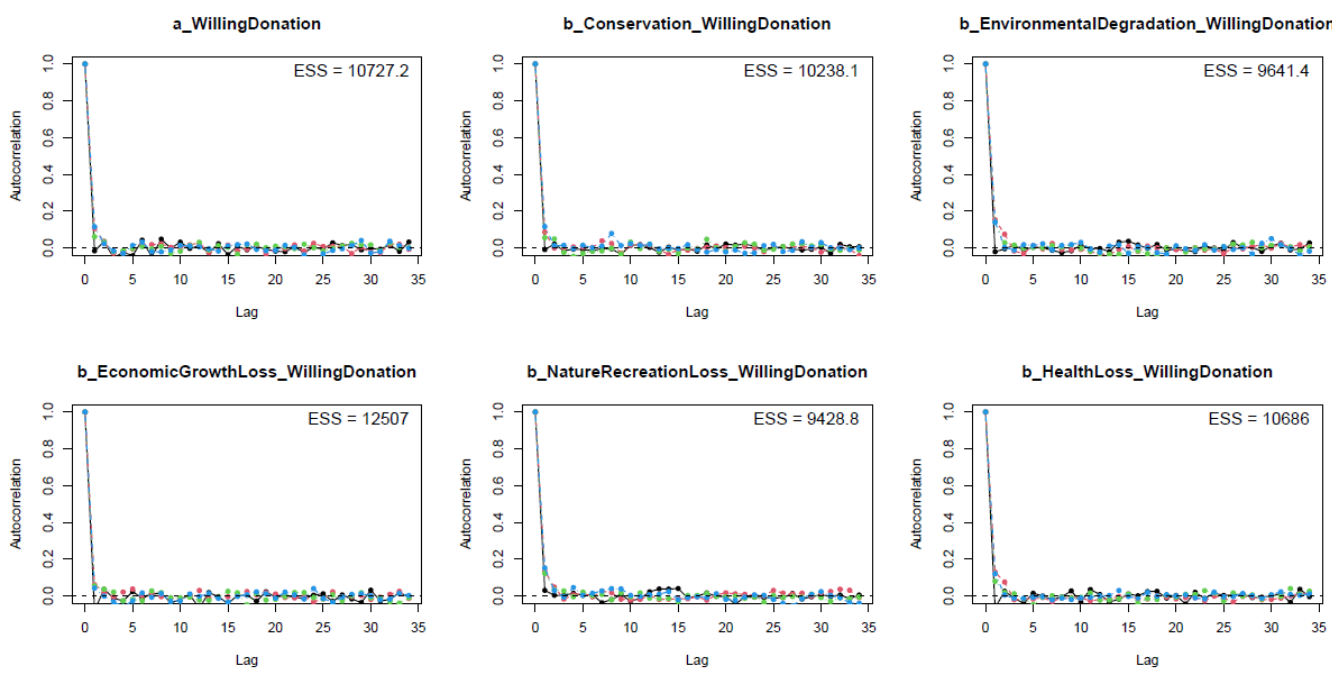

b_KnowledgeLoss_WillingDonation

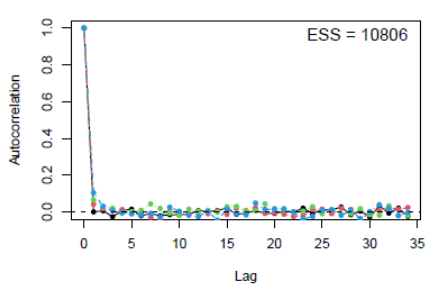

Figure A12: Model 3b's autocorrelation plots with priors as norm $(1,0.5)$ 


\section{References}

1. Steffen, W.; Richardson, K.; Rockström, J.; Cornell, S.E.; Fetzer, I.; Bennett, E.M.; Biggs, R.; Carpenter, S.R.; De Vries, W.; De Wit, C.A. Planetary boundaries: Guiding human development on a changing planet. Science 2015, 347.

2. Intergovernmental Science-Policy Platform on Biodiversity and Ecosystem Services. Nature's Dangerous Decline 'Unprecedented' Species Extinction Rates 'Accelerating'. Available online: https://www.unenvironment.org/news-and-stories/press-release/naturesdangerous-decline-unprecedented-species-extinction-rates (accessed on 24/06).

3. World Wildlife Fund. Living Planet Report 2020 - Bending the curve of biodiversity loss; Gland, Switzerland, 2020.

4. Emerton, L.; Bishop, J.; Thomas, L. Sustainable Financing of Protected Areas: A global review of challenges and options; IUCN: 2006.

5. Thur, S.M. User fees as sustainable financing mechanisms for marine protected areas: An application to the Bonaire National Marine Park. Marine policy 2010, 34, 63-69.

6. Dharmaratne, G.S.; Sang, F.Y.; Walling, L.J. Tourism potentials for financing protected areas. Annals of Tourism Research 2000, 27, 590-610.

7. Watson, J.E.; Dudley, N.; Segan, D.B.; Hockings, M. The performance and potential of protected areas. Nature 2014, 515, 67-73.

8. UNEP-WCMC; IUCN. Protected Planet Report 2020; Cambridge, UK; Gland, Switzerland, 2021.

9. Bovarnick, A.; Fernandez-Baca, J.; Galindo, J.; Negret, H. Financial sustainability of protected areas in Latin America and the Caribbean: investment policy guidance; United Nations Development Programme (UNDP) and The Nature Conservancy (TNC): 2010.

10. Bui, H.T.; Pham, L.H.; Jones, T.E. Governance and Management of Protected Areas in Vietnam: Nature-Based Tourism in Mountain Areas. In Nature-Based Tourism in Asia's Mountainous Protected Areas: A Trans-regional Review of Peaks and Parks, Jones, T.E., Bui, H.T., Apollo, M., Eds.; Springer: Cham, Switzerland, 2021; pp. 173-195.

11. Jones, T.E.; Apollo, M.; Bui, H.T. Mountainous Protected Areas \& Nature-Based Tourism in Asia. In Nature-Based Tourism in Asia's Mountainous Protected Areas, Jones, T.E., Bui, H.T., Apollo, M., Eds.; Springer: Cham, 2021; pp. 3-15.

12. Whitelaw, P.A.; King, B.E.; Tolkach, D. Protected areas, conservation and tourismfinancing the sustainable dream. Journal of Sustainable Tourism 2014, 22, 584-603.

13. Balmford, A.; Green, J.M.; Anderson, M.; Beresford, J.; Huang, C.; Naidoo, R.; Walpole, M.; Manica, A. Walk on the wild side: estimating the global magnitude of visits to protected areas. PLoS Biol 2015, 13, e1002074.

14. Mansourian, S.; Dudley, N. Public funds to protected areas; WWF International: Gland, Switzerland, 2008.

15. Naughton-Treves, L.; Holland, M.B.; Brandon, K. The role of protected areas in conserving biodiversity and sustaining local livelihoods. Annu. Rev. Environ. Resour. 2005, 30, 219252.

16. Walpole, M.J.; Goodwin, H.J. Local attitudes towards conservation and tourism around Komodo National Park, Indonesia. Environmental conservation 2001, 160-166.

17. World Bank. Banking on Protected Areas : Promoting Sustainable Protected Area Tourism to Benefit Local Economies; Washington, DC., 2021. 
18. Wang, P.-W.; Jia, J.-B. Tourists' willingness to pay for biodiversity conservation and environment protection, Dalai Lake protected area: Implications for entrance fee and sustainable management. Ocean and Coastal Management 2012, 62, 24-33.

19. Bhandari, A.K.; Heshmati, A. Willingness to pay for biodiversity conservation. Journal of Travel and Tourism Marketing 2010, 27, 612-623.

20. Estifanos, T.; Polyakov, M.; Pandit, R.; Hailu, A.; Burton, M. What are tourists willing to pay for securing the survival of a flagship species? The case of protection of the Ethiopian wolf. Tourism Economics 2021, 27, 45-69.

21. Baral, N.; Dhungana, A. Diversifying finance mechanisms for protected areas capitalizing on untapped revenues. Forest Policy and Economics 2014, 41, 60-67.

22. Gelcich, S.; Amar, F.; Valdebenito, A.; Castilla, J.C.; Fernandez, M.; Godoy, C.; Biggs, D. Financing marine protected areas through visitor fees: Insights from tourists willingness to pay in Chile. Ambio 2013, 42, 975-984.

23. Fredman, P.; Tyrväinen, L. Frontiers in nature-based tourism. Scandinavian Journal of Hospitality and Tourism 2010, 10, 177-189.

24. Lundmark, L.; Müller, D.K. The supply of nature-based tourism activities in Sweden. Tourism: An International Interdisciplinary Journal 2010, 58, 379-393.

25. Frost, W.; Laing, J.; Beeton, S. The future of nature-based tourism in the Asia-Pacific region. Journal of Travel Research 2014, 53, 721-732.

26. Jones, T.E.; Nguyen, M.-H. Nature-Based Tourism Motivations and Visit Profiles of Domestic and International Segments to a Japanese National Park. Quaestiones Geographicae 2021, 40, 77-92.

27. Vuong, Q.H. The semiconducting principle of monetary and environmental values exchange. Economics and Business Letters 2021, 10, 284-290, doi:10.17811/ebl.10.3.2021.284-290.

28. Vuong, Q.-H. Western monopoly of climate science is creating an eco-deficit culture. Economy, Land \& Climate Insight 2021.

29. Harrison, L.E. Culture matters. The National Interest 2000, 60, 55-65.

30. Nguyen, M.-H.; Jones, T.E. Predictors of support for a biodiversity loss countermeasure and bushmeat consumption among Vietnamese urban residents. OSF Preprints 2021, doi:10.31219/osf.io/9kdbw.

31. Nguyen, M.-H. Multifaceted interactions between urban humans and biodiversity-related concepts: A developing-country dataset. Data Intelligence 2021, 3, 578-605.

32. Creswell, J.W.; Poth, C.N. Qualitative inquiry and research design: choosing among five approaches; SAGE: Los Angeles, 2018.

33. Nguyen, M.-H.; Le, T.-T. Bayesian Mindsponge Framework. Scholarly Community Encyclopedia 2021.

34. Nguyen, M.-H.; Le, T.-T.; Ho, M.-T.; Nguyen, H.T.T.; Vuong, Q.-H. Alice in Suicideland: Exploring the Suicidal Ideation Mechanism through the Sense of Connectedness and HelpSeeking Behaviors. IJERPH 2021, 18, 3681, doi:https://doi.org/10.3390/ijerph18073681.

35. Vuong, Q.-H.; Nguyen, M.-H.; Le, T.-T. A mindsponge-based investigation into the psycho-religious mechanism behind suicide attacks; De Gruyter / Sciendo: Warsaw, Poland, 2021.

36. Vuong, Q.-H.; Nguyen, M.-H.; Le, T.-T. Home scholarly culture, book selection reason, and academic performance: Pathways to book reading interest among secondary school 
students. European Journal of Investigation in Health, Psychology and Education 2021, 11, 468-495, doi:https://doi.org/10.3390/ejihpe11020034.

37. Vuong, Q.-H.; Napier, N.K. Acculturation and global mindsponge: an emerging market perspective. International Journal of Intercultural Relations 2015, 49, 354-367.

38. Vuong, Q.-H. Global Mindset as the Integration of Emerging Socio-Cultural Values Through Mindsponge Processes: A Transition Economy Perspective. In Global Mindsets: Exploration and Perspectives, Kuada, J., Ed.; Routledge: 2016; pp. 109-126.

39. Csilléry, K.; Blum, M.G.; Gaggiotti, O.E.; François, O. Approximate Bayesian computation (ABC) in practice. Trends in Ecology and Evolution 2010, 25, 410-418, doi:10.1016/j.tree.2010.04.001.

40. Kerr, N.L. HARKing: Hypothesizing after the results are known. Personality Social Psychology Review 1998, 2, 196-217.

41. Vuong, Q.H.; Ho, M.T.; La, V.P. 'Stargazing' and p-hacking behaviours in social sciences: some insights from a developing country. European Science Editing 2019, 45, 54-55.

42. Depaoli, S.; Van de Schoot, R. Improving transparency and replication in Bayesian statistics: The WAMBS-Checklist. Psychological Methods 2017, 22, 240.

43. Block, J.H.; Wagner, M.J.B.S.; Environment, t. The effect of family ownership on different dimensions of corporate social responsibility: Evidence from large US firms. Business Strategy and the Environment 2014, 23, 475-492.

44. Hahn, E.D.; Doh, J.P. Using Bayesian methods in strategy research: an extension of Hansenet al. Strategic Management Journal 2006, 27, 783-798.

45. Baker, M. Over half of psychology studies fail reproducibility test. Nature 2015, doi:https://doi.org/10.1038/nature.2015.18248.

46. Open Science Collaboration. Estimating the reproducibility of psychological science. Science 2015, 349, doi:10.1126/science.aac4716.

47. Halsey, L.G.; Curran-Everett, D.; Vowler, S.L.; Drummond, G.B. The fickle P value generates irreproducible results. Nature methods 2015, 12, 179-185.

48. Leamer, E.E.J.T.r.o.e.; statistics. Multicollinearity: a Bayesian interpretation. The Review of Economics and Statistics 1973, 55, 371-380.

49. Adepoju, A.A.; Ojo, O.O. Bayesian method for solving the problem of multicollinearity in regression. Afrika Statistika 2018, 13, 1823-1834.

50. Jaya, I.; Tantular, B.; Andriyana, Y. A Bayesian approach on multicollinearity problem with an Informative Prior. In Proceedings of the Journal of Physics: Conference Series, 2019; p. 012021.

51. Vuong, Q.H.; Ho, M.-T.; Nguyen, M.-H.; Thang Hang, P.; Vuong, T.-T.; Khuc, Q.; Ho, H.-A.; La, V.-P. On the environment-destructive probabilistic trends: a perceptual and behavioral study on video game players. Technology in Society 2021, 65, 101530, doi:10.1016/j.techsoc.2021.101530.

52. La, V.-P.; Vuong, Q.-H. bayesvl: Visually learning the graphical structure of Bayesian networks and performing MCMC with'Stan'. The Comprehensive $R$ Archive Network (CRAN) 2019.

53. Vuong, Q.-H.; La, V.-P.; Nguyen, M.-H.; Ho, M.-T.; Ho, M.-T.; Mantello, P. Improving Bayesian statistics understanding in the age of Big Data with the bayesvl $\mathrm{R}$ package. Software Impacts 2020, 4, 100016, doi:10.1016/j.simpa.2020.100016. 
54. Vuong, Q.-H.; La, V.-P.; Nguyen, M.-H.; Ho, M.-T.; Tran, T.; Ho, M.-T. Bayesian analysis for social data: A step-by-step protocol and interpretation. MethodsX 2020, 7, 100924, doi:10.1016/j.mex.2020.100924.

55. Vehtari, A.; Gabry, J. Bayesian Stacking and Pseudo-BMA weights using the loo package, loo 2.2.0; 2019.

56. Haq, G.; Cambridge, H.; Owen, A. A targeted social marketing approach for community pro-environmental behavioural change. Local Environment 2013, 18, 1134-1152.

57. Veríssimo, D. The past, present, and future of using social marketing to conserve biodiversity. Social Marketing Quarterly 2019, 25, 3-8.

58. Veríssimo, D.; Bianchessi, A.; Arrivillaga, A.; Cadiz, F.C.; Mancao, R.; Green, K. Does it work for biodiversity? Experiences and challenges in the evaluation of social marketing campaigns. Social Marketing Quarterly 2018, 24, 18-34.

59. Ryan, J.; Mellish, S.; Dorrian, J.; Winefield, T.; Litchfield, C. Effectiveness of biodiversity-conservation marketing. Conservation Biology 2020, 34, 354-367.

60. Marion, J.L.; Reid, S.E. Minimising visitor impacts to protected areas: The efficacy of low impact education programmes. Journal of Sustainable Tourism 2007, 15, 5-27.

61. Davis, E.O.; Willemsen, M.; Dang, V.; O’Connor, D.; Glikman, J.A. An updated analysis of the consumption of tiger products in urban Vietnam. Global Ecology and Conservation 2020, 22, e00960.

62. Davis, E.O.; Glikman, J.A.; Crudge, B.; Dang, V.; Willemsen, M.; Nguyen, T.; O'Connor, D.; Bendixsen, T. Consumer demand and traditional medicine prescription of bear products in Vietnam. Biological Conservation 2019, 235, 119-127.

63. Sexton, R.; Nguyen, T.; Roberts, D.L. The use and prescription of pangolin in traditional Vietnamese medicine. Tropical Conservation Science 2021, 14, 1940082920985755.

64. Mace, G.M.; Norris, K.; Fitter, A.H. Biodiversity and ecosystem services: a multilayered relationship. Trends in Ecology \& Evolution 2012, 27, 19-26.

65. Nguyen, M.-H.; Jones, T.E. Exploring biodiversity mental constructs of urban residents in an emerging South-East Asian country. OSF Preprints 2021, doi:10.31219/osf.io/kyc4v.

66. Bakhtiari, F.; Jacobsen, J.B.; Strange, N.; Helles, F. Revealing lay people's perceptions of forest biodiversity value components and their application in valuation method. Global Ecology Conservation 2014, 1, 27-42.

67. Vuong, Q.-H. Reform retractions to make them more transparent. Nature 2020, 582, 149, doi:10.1038/d41586-020-01694-x. 